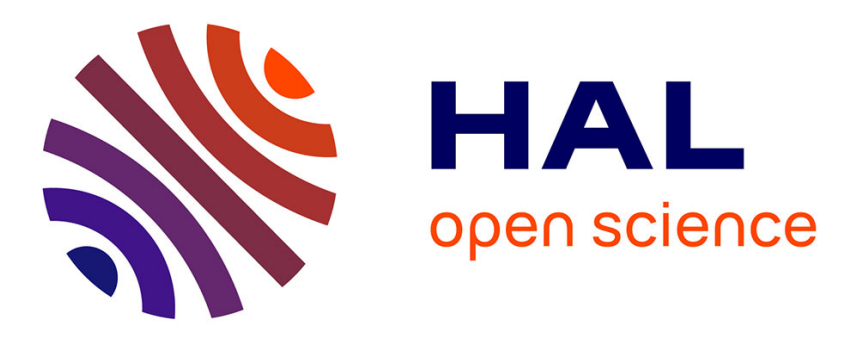

\title{
Exact Solutions for the Vibration of Finite Granular Beam Using Discrete and Gradient Elasticity Cosserat Models
}

Sina Massoumi, Noël Challamel, Jean Lerbet

\section{- To cite this version:}

Sina Massoumi, Noël Challamel, Jean Lerbet. Exact Solutions for the Vibration of Finite Granular Beam Using Discrete and Gradient Elasticity Cosserat Models. Journal of Sound and Vibration, 2020. hal-03241669

\section{HAL Id: hal-03241669 \\ https://hal.science/hal-03241669}

Submitted on 28 May 2021

HAL is a multi-disciplinary open access archive for the deposit and dissemination of scientific research documents, whether they are published or not. The documents may come from teaching and research institutions in France or abroad, or from public or private research centers.
L'archive ouverte pluridisciplinaire HAL, est destinée au dépôt et à la diffusion de documents scientifiques de niveau recherche, publiés ou non, émanant des établissements d'enseignement et de recherche français ou étrangers, des laboratoires publics ou privés. 


\title{
Exact Solutions for the Vibration of Finite Granular Beam Using Discrete and Gradient Elasticity Cosserat Models
}

\author{
Sina Massoumi ${ }^{a}$, Noël Challamel ${ }^{b, *}$, Jean Lerbet ${ }^{a}$ \\ a Univ. Evry - Université Paris-Saclay, Laboratoire de Mathématiques et Modélisation d’Evry, \\ LaMME -UMR CNRS 8071, 23 bvd de France, 91037 Evry - France \\ b Univ. Bretagne Sud, IRDL - UBS - UMR CNRS 6027, Centre de Recherche, Rue de Saint Maudé \\ - BP 92116, 56321 Lorient cedex - France
}

\begin{abstract}
The present study theoretically investigates the free vibration problem of a discrete granular system. This problem can be considered as a simple model to rigorously study the effects of the microstructure on the dynamic behavior of the equivalent continuum structural model. The model consists of uniform grains confined by discrete elastic interactions, to take into account the lateral granular contributions. This repetitive discrete system can be referred to discrete Cosserat chain or a lattice elastic model with shear interaction. First for the simply supported granular beam resting on Winkler foundations, due to the critical frequencies which concern the nature of the dynamic results, the natural frequencies are exactly calculated, starting from the resolution of the linear difference eigenvalue problem. The natural frequencies of such a granular model are analytically calculated for whatever modes. It is shown that the difference equations governed to the discrete system converge to the differential equations of the Bresse-Timoshenko beam resting on Winkler foundation (also classified as a continuous Cosserat beam model) for an infinite number of grains. A gradient Bresse-Timoshenko model is constructed from continualization of the difference equations. This continuous gradient elasticity Cosserat model is obtained from a polynomial or a rational expansion of the pseudo-differential operators, stemming from the continualization process. Scale effects of the granular chain are captured by the continuous gradient elasticity model. The natural frequencies of the continuous gradient Cosserat models are compared with those of the discrete Cosserat model associated with the granular chain. The results clarify the dependency of the beam dynamic responses to the beam length ratio.
\end{abstract}

Keywords: Granular medium; Cosserat continuum; Discrete Cosserat formulation; Gradient elasticity; Timoshenko beam.

* Corresponding author. E-mail address: noel.challamel@univ-ubs.fr. 


\section{Introduction}

In order to adapt a standard continuum theory to granular materials, it is necessary to introduce the independent rotational degrees of freedom (DOF) in addition to the conventional translational ones. This helps to describe accurately the relative movements between the microstructure and the average macroscopic deformations. One may obtain higher-order gradient continua with additional degrees of freedom. One may also obtain Cosserat modeling that consequently leads to non-classical continuum or polar continuum theories (Cosserat type theories, e.g. Cosserat and Cosserat [1]; Nowacki [2]). Voigt [3] was the pioneer of developing this concept who first showed the existence of couple -stress in materials. Cosserat continuum theories belong to the larger class of generalized continua which introduce intrinsic length scales into continuum mechanics via higher-order gradients or additional degrees of freedom (Eringen [4, 5], Forest [6]). Feng [7] analyzed the behavior of the granular medium considering normal, shear and rotation interactions. In contrast, the classical continuum mechanics ignores the rotational interactions among particles and neglects the size effect of material particles. Schwartz et al. [8] studied dispersive analysis of a granular medium with normal and shear interactions neglecting the rotation effects.

On the other hand, in beam analysis, the Bresse-Timoshenko model takes into account both beam shear flexibility and rotatory inertia (Bresse [9] and Timoshenko [10, 11]). The effects of shear and rotational inertia can be significant in case of calculating eigenfrequencies for short beams, or in case of sufficiently small shear modulus. The Bresse-Timoshenko beam model is also a generalization of the Euler-Bernoulli model and admits a kinematics with two independent fields, a field of transverse displacement and a field of rotation. Timoshenko pointed out that the effects of cross-sectional dimensions on the beam dynamic behavior and frequencies could be significant. Timoshenko $[10,11]$ calculated the exact eigenfrequencies for such a beam with two degrees of freedom resting on two simple supports. Several lattice models have been developed based on microstructured Timoshenko in order to go further in understanding the structure behavior (see Ostoja-Starzewski [12] and Attar et al. [13]). The static and dynamic properties of a Cosserat-type lattice interface studied by Vasiliev et al. [14]. Calculation of eigenfrequencies for a Bresse-Timoshenko beam with any boundary conditions and elastic interaction with a rigid medium is obtained by Wang and Stephens [15], Manevich [16] or Elishakoff et al. [17] (see more recently Elishakoff [18] and Challamel and Elishakoff [19]). Bresse-Timoshenko beam theory is merely a one-dimensional Cosserat continuum medium by considering two independent translational and rotational degrees of freedom (Rubin [20] and Exadaktylos [21]). Thus, there is a fundamental link between these two continuum theories. In this paper, it is noticed the relation between Coasserat discrete theories and the continuum ones.

The present study focuses on the vibration of a granular beam with both bending and shear granular interactions. The granular beam is assumed to interact elastically with a rigid elastic support, a discrete elastic foundation labeled as a discrete Winkler foundation (Winkler [22]). Note that the difference equations governed to the model coincide with the ones of Cosserat granular model of Pasternak and Mühlhaus [23] in the absence of an elastic foundation, but differ from the ones of the discrete shear model studied by Duan et al. [24]. However, for some specific bending/ shear interaction modeling, the model developed by Bacigalupo and Gambarotta [25] can be mathematically reformulated with the difference equation presented in this paper.

This paper is arranged as follows: First, a discrete granular beam model is introduced from a geometrical and a mechanical point of view. The grain interaction and material parameters are defined in detail. Then from the dynamic analysis of the lattice beam model, the deflection 
equations of the finite granular beam are derived. This fourth-order linear difference equation is solved by using the exact resolution of the difference equation. For an infinite number of grains, the deflection equation of a continuous beam (a fourth-order linear differential equation) is obtained asymptotically. Next, the eigenfrequencies of the discrete granular model and the continuous one, are obtained and compared as well. In the end, two asymptotic continualization methods are used to investigate continuous beam from the discrete lattice problem. With this aim, the polynomial expansion of Taylor and the rational expansion of Padé (with involved pseudo-differential operators) are used to derive new enriched beam models. These two nonlocal continualization approaches with the introduction of the gradient terms engage the neighbor influences which allow the passage from discrete result to continuous ones and simultaneously capture the length effect.

\section{Granular Model}

A granular beam of length $L$ resting on two simple supports is modeled by a finite number of grains interacting together. Such a model could be presented by considering the microstructured granular chain comprising $n+1$ rigid grains with diameter $a(a=L / n)$ that are connected by $n$ shear and rotational springs, as shown in Figure 1. It is assumed that the elastic support springs are located at the center of each rigid grain. Each grain has two degrees of freedom which are denoted by $W_{i}$ for the deflection and $\Theta_{i}$ for the rotation. This model is slightly different from the one of Challamel et al. [26] where the nodal kinematics and the Winkler elastic foundation are located at the grain interface. The aim of this paper consists in finding the vibration equation of this granular chain and then trying to obtain the natural frequencies.

(a)

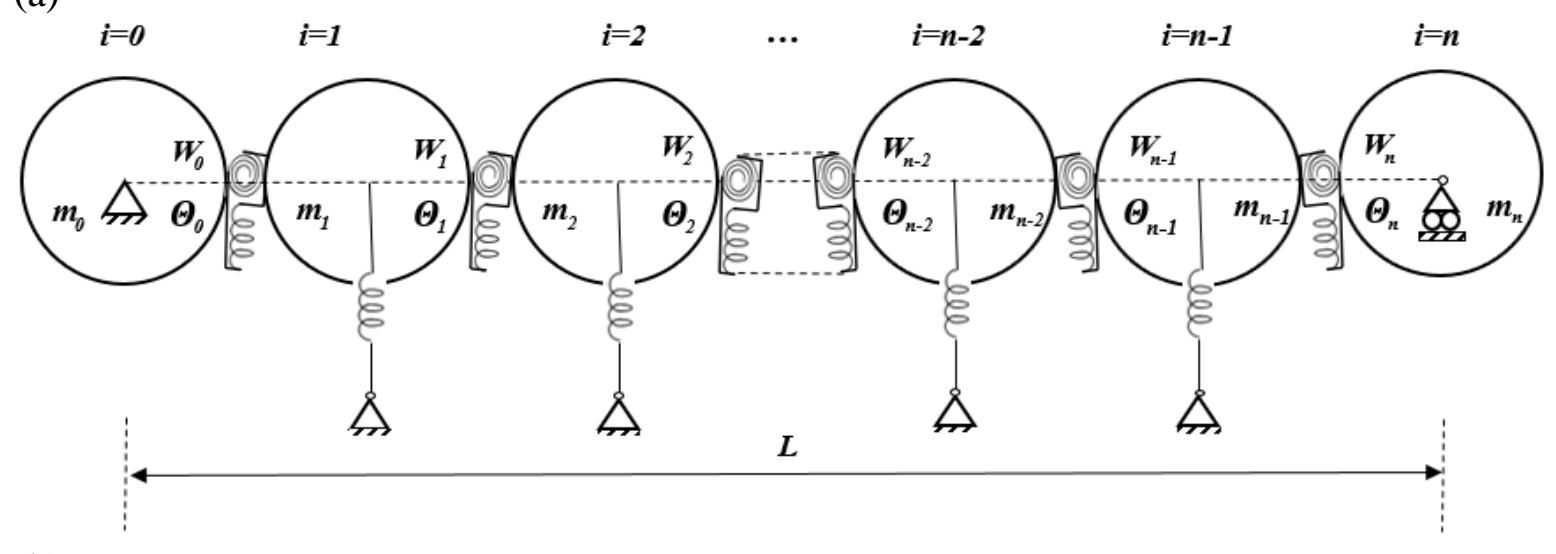

(b)

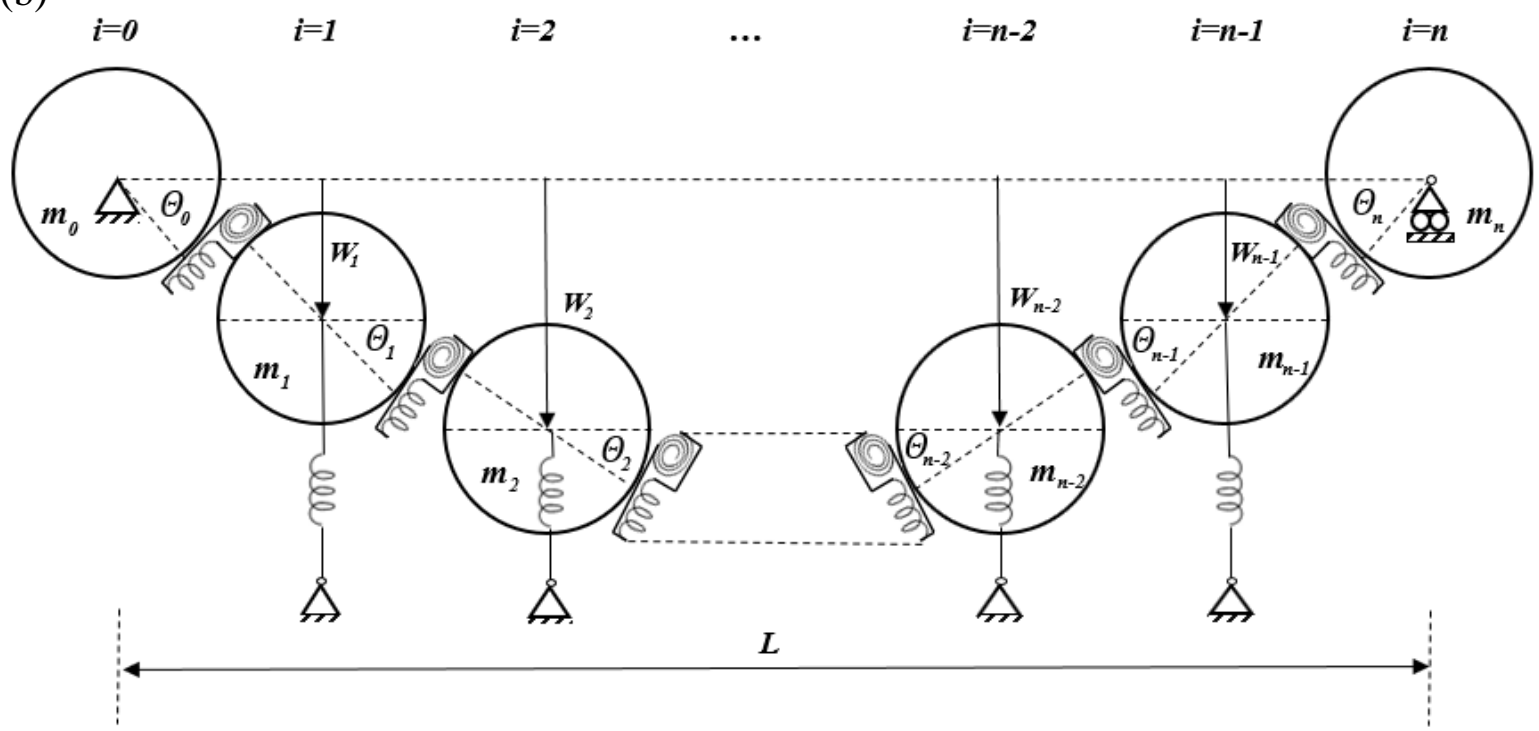


Fig. 1. A discrete shear granular chain model composed of $n+1$ grain; (a) undeformed and (b) deformed.

The total kinetic energy of the model may be expressed as follows:

$$
T=\frac{1}{2} \sum_{i=0}^{n} m_{i} \dot{W}_{i}^{2}+\frac{1}{2} \sum_{i=0}^{n} I_{m_{i}} \dot{\Theta}_{i}^{2}
$$

where for $i$ in [1, n-1], $I_{m_{i}}=\frac{\rho I L}{n}=\rho I a$ is the second moment of inertia of the beam segment and $m_{i}$ is the mass term for each grain that is defined for the inter grains by $m_{i}=\rho a$.

The strain energy function due to deformed shear spring (shear term) is given by

$$
U_{s}=\frac{1}{2} \sum_{i=0}^{n-1} S\left(W_{i+1}-W_{i}-a \frac{\Theta_{i+1}+\Theta_{i}}{2}\right)^{2}
$$

where $S$ is the shear stiffness which can be expressed with respect to the shear stiffness $K_{S} G A$ of the equivalent beam. The shear stiffness parameter could be defined as $S=\frac{K_{S} G A}{a}=\frac{n K_{S} G A}{L}$ in which $G$ is the shear modulus, $A$ is the cross-sectional area of the beam and $K_{s}$ is an equivalent shear correction coefficient.

In the present formulation, the kinematic variables are measured at nodes $i$ located at the center of each grain, which is consistent with the approach followed for instance by Pasternak and Mühlhaus [23].

The strain energy function due to deformed rotational springs (bending term) may be obtained by

$$
\mathrm{U}_{\mathrm{b}}=\frac{1}{2} \sum_{\mathrm{i}=0}^{\mathrm{n}-1} \mathrm{C}\left(\Theta_{\mathrm{i}+1}-\Theta_{\mathrm{i}}\right)^{2}
$$

where $C$ is the rotational stiffness located at the connection between each grain. This discrete stiffness can be expressed with respect to the bending stiffness $E I$ of the equivalent beam and thus would be defined as $C=\frac{E I}{a}=\frac{n E I}{L}$. where $E$ is Young's modulus and $I$ is the second moment of area.

The elastic energy in the discrete elastic support (Winkler [22]) is given by

$$
U_{\text {Winkler }}=\frac{1}{2} \sum_{i=0}^{n} K W_{i}^{2}
$$

where $K=k a$ is the discrete stiffness of the elastic support and is attached to the center of each grain.

The Lagrangian of the system may be defined as $L=T-\left(U_{S}+U_{b}+U_{\text {Winkler }}\right)$ which slightly differs from the shear lattice model considered by Ostoja-Starzewski [12] for the shear term. By substituting the kinetic and potential terms, the Lagrangian may be expressed as:

$$
\begin{aligned}
& L=\left[\frac{1}{2} \sum_{i=0}^{n} m_{i} \dot{W}_{i}^{2}+\frac{1}{2} \sum_{i=0}^{n} I_{m_{i}} \dot{\Theta}_{i}^{2}\right]-\left[\frac{1}{2} \sum_{i=0}^{n-1} S\left(W_{i+1}-W_{i}-a \frac{\Theta_{i+1}+\theta_{i}}{2}\right)^{2}+\frac{1}{2} \sum_{i=0}^{n-1} C\left(\Theta_{i+1}-\Theta_{i}\right)^{2}+\right. \\
& \left.\frac{1}{2} \sum_{i=0}^{n} K W_{i}^{2}\right]
\end{aligned}
$$

The system of difference equations for both the discrete displacement and rotation fields is obtained from application of Hamilton's principle, given by: 


$$
\int_{t_{1}}^{t_{2}} \delta L d t=\int_{t_{1}}^{t_{2}}(\delta T-\delta U) d t=0
$$

Using Eq. (6) based on the energy function of Eq. (5) leads to the following difference equation system

$$
\left\{\begin{array}{c}
S\left(W_{i+1}+W_{i-1}-2 W_{i}\right)-\frac{a}{2} S\left(\Theta_{i+1}-\Theta_{i-1}\right)-k a W_{i}-m_{i} \ddot{W}_{i}=0 \quad(i=1, \ldots, n-1) \\
C\left(\Theta_{i+1}+\Theta_{i-1}-2 \Theta_{i}\right)+\frac{a}{2} S\left(W_{i+1}-W_{i-1}\right)-\frac{a^{2}}{4} S\left(\Theta_{i+1}+\Theta_{i-1}+2 \Theta_{i}\right)-I_{m_{i}} \ddot{\Theta}_{i}=0 \quad(i=1, \ldots, n-1)
\end{array}\right.
$$

where for the variationally-based boundary conditions, the following four equations could be obtained

$$
\left\{\begin{array}{c}
{\left[S\left(W_{1}-W_{0}-\frac{a}{2}\left(\Theta_{1}+\Theta_{0}\right)\right)-k a W_{0}-m_{0} \ddot{W}_{0}\right] \delta W_{0}=0} \\
{\left[S\left(W_{n}-W_{n-1}-\frac{a}{2}\left(\Theta_{n}+\Theta_{n-1}\right)\right)+k a W_{n}+m_{n} \ddot{W}_{n}\right] \delta W_{n}=0} \\
{\left[C\left(\Theta_{1}-\Theta_{0}\right)+\frac{a}{2} S\left(W_{1}-W_{0}\right)-\frac{a^{2}}{4} S\left(\Theta_{1}+\Theta_{0}\right)-I_{m_{0}} \ddot{\Theta}_{0}\right] \delta \Theta_{0}=0} \\
{\left[-C\left(\Theta_{n-1}-\Theta_{n}\right)-\frac{a}{2} S\left(W_{n}-W_{n-1}\right)+\frac{a^{2}}{4} S\left(\Theta_{n}+\Theta_{n-1}\right)-I_{m_{n}} \ddot{\Theta}_{n}\right] \delta \Theta_{n}=0}
\end{array}\right.
$$

Introducing the following pseudo-difference operators, Eq. (7) might be generalized and rewritten compactly by Eq. (9).

$$
\begin{gathered}
\delta_{0} W_{i}=\frac{W_{i+1}+2 W_{i}+W_{i-1}}{4}, \delta_{1} W_{i}=\frac{W_{i+1}-W_{i-1}}{2 a}, \delta_{2} W_{i}=\frac{W_{i+1}-2 W_{i}+W_{i-1}}{a^{2}} \\
K_{S} G A\left(\delta_{2} W_{i}\right)-K_{s} G A\left(\delta_{1} \Theta_{i}\right)-k W_{i}-\rho A \ddot{W}_{i}=0 \\
E I\left(\delta_{2} \Theta_{i}\right)+K_{S} G A\left(\delta_{1} W_{i}\right)-K_{S} G A\left(\delta_{0} \Theta_{i}\right)-\rho I \ddot{\Theta}_{\imath}=0
\end{gathered}
$$

Assuming a harmonic motion $W_{i}=w_{i} e^{j \omega t}$ and $\Theta_{i}=\theta_{i} e^{j \omega t}$ with $j^{2}=-1$, Eq. (10) may be written in a matrix form

$$
\left(\begin{array}{cc}
E I \delta_{2}-K_{S} G A \delta_{0}+\rho I \omega^{2} & K_{S} G A \delta_{1} \\
K_{S} G A \delta_{1} & k-K_{S} G A \delta_{2}-\rho A \omega^{2}
\end{array}\right)\left(\begin{array}{c}
\theta \\
w
\end{array}\right)_{i}=\left(\begin{array}{l}
0 \\
0
\end{array}\right)
$$

These difference equations system (11) have been obtained by Pasternak and Mühlhaus [23] neglecting the elastic Winkler foundation $(k=0)$. With consideration of an infinite number of grains $(n \rightarrow \infty)$ referring to the continuum beam, it converges to the coupled system differential equations Eq. (12) which has been obtained by Bresse [9] and Timoshenko [10,11] in the absence of a Winkler foundation $(k=0)$ and assuming the shear correction factor to be unity $\left(K_{s}=1\right)$. Eq. (12) valid for a Bresse-Timoshenko beam on elastic foundation have been also obtained by Wang and Stephens [15] and Manevich [16].

$$
\left(\begin{array}{cc}
E I \partial_{x}^{2}-K_{s} G A+\rho I \omega^{2} & K_{s} G A \partial_{x} \\
K_{S} G A \partial_{x} & k-K_{S} G A \partial_{x}^{2}-\rho A \omega^{2}
\end{array}\right)\left(\begin{array}{c}
\theta \\
w
\end{array}\right)=\left(\begin{array}{l}
0 \\
0
\end{array}\right)
$$

It is possible to introduce the following pseudo-differential operators

$$
\delta_{0}=\frac{e^{a \partial_{x}}+2+e^{-a \partial_{x}}}{4}=\cosh ^{2}\left(\frac{a \partial_{x}}{2}\right)
$$




$$
\begin{gathered}
\delta_{1}=\frac{e^{a \partial_{x}}-e^{-a \partial_{x}}}{2 a}=\frac{\sinh \left(a \partial_{x}\right)}{a} \\
\delta_{2}=\frac{e^{a \partial_{x}}-2+e^{-a \partial_{x}}}{a^{2}}=\frac{4}{a^{2}} \sinh ^{2}\left(\frac{a \partial_{x}}{2}\right)
\end{gathered}
$$

The relation could be obtained between these operators as

$$
\delta_{2} \delta_{0}=\delta_{0} \delta_{2}=\delta_{1}{ }^{2}
$$

The same relations could be obtained for the pseudo-difference operators of Eq. (9). Going back to the discrete granular beam model Eq. (11), this characteristic equation has nontrivial solutions only if the determinant of the matrix is zero. Using the property of Eq. (14) gives the fourth-order difference equation for the deflection as follows:

$$
\begin{aligned}
& {\left[E I \delta_{2}{ }^{2}+\left(\rho I \omega^{2}-\frac{k E I}{K_{S} G A}+\frac{E I \rho \omega^{2}}{K_{s} G}\right) \delta_{2}+\left(k-\rho A \omega^{2}\right) \delta_{0}-\frac{k \rho I \omega^{2}}{K_{s} G A}+\frac{\rho^{2} I \omega^{4}}{K_{s} G}\right] w_{i}=0} \\
& {\left[E I \delta_{2}{ }^{2}+\left(\rho I \omega^{2}-\frac{k E I}{K_{s} G A}+\frac{E I \rho \omega^{2}}{K_{s} G}\right) \delta_{2}+\left(k-\rho A \omega^{2}\right) \delta_{0}-\frac{k \rho I \omega^{2}}{K_{s} G A}+\frac{\rho^{2} I \omega^{4}}{K_{s} G}\right] \theta_{i}=0}
\end{aligned}
$$

Neglecting the Winkler elastic foundation $(k=0)$, Eq. (15) leads to

$$
\left[\delta_{2}{ }^{2}+\omega^{2}\left(\frac{\rho}{E}+\frac{\rho}{K_{S} G}\right) \delta_{2}-\omega^{2}\left(\frac{\rho A}{E I} \delta_{0}-\frac{\rho^{2} \omega^{2}}{E K_{S} G}\right)\right] w_{i}=0
$$

Duan et al. [24] also studied a discrete Timoshenko beam model based on rigid links where the displacement fields are defined at the joint element. The scheme of their study slightly differs from the granular model considered in this paper, essentially from the last term in the fourthorder difference equation of each model. The appearance of pseudo-difference operator $\delta_{0}$ in this model in comparison with Duan et al. [24], stems from the enhanced shear interaction modeling of the granular elements which refers to the fundamental difference of the two microstructural models.

$$
\left[\delta_{2}^{2}+\omega^{2}\left(\frac{\rho}{E}+\frac{\rho}{K_{S} G}\right) \delta_{2}-\omega^{2}\left(\frac{\rho A}{E I}-\frac{\rho^{2} \omega^{2}}{E K_{s} G}\right)\right] w_{i}=0
$$

Eq. (17) and Eq. (18) are the governing deflection equations of two alternative discrete granular models for studying the beam vibration and its dynamic responses.

The fourth-order difference equation Eq. (15) is equivalent to the one of Challamel et al. [27] in the static range $(\omega=0)$. Considering infinite number of grains $(n \rightarrow \infty)$ for the continuum beam, the fourth-order differential equation valid for a Bresse-Timoshenko beam on Winkler elastic foundation is given by Eq. (19) which also could be compared well by Wang and Stephens [15], Cheng and Pantelides [28] and Manevich [16].

$$
\left.\frac{d^{4} w}{d x^{4}}+\left(\frac{\rho \omega^{2}}{E}\left(1+\frac{E}{k_{S} G}\right)-\frac{k}{k_{S} G A}\right) \frac{d^{2} w}{d x^{2}}-\left(\frac{\rho \omega^{2}}{E}\left(\frac{A}{I}+\frac{k}{k_{S} G A}-\frac{\rho \omega^{2}}{k_{S} G}\right)-\frac{k}{E I}\right)\right] w=0
$$

\section{Exact Solution}

\subsection{Resolution of The Difference Equation}

In this section, the exact solution for the fourth-order linear difference eigenvalue problem of Eq. (15) will be established (see the books of Goldberg [29] or Elaydi [30] for the general solution of linear difference equations). This approach, as detailed for instance by Elishakoff 
and Santoro [31, 32], has been used to analyze the error in the finite difference based probabilistic dynamic problems. Eq. (15) and Eq. (16) restricted to the vibration terms, the linear fourth-order difference equation may be expanded as

$$
\begin{gathered}
\left(w_{i+2}-4 w_{i+1}+6 w_{i}-4 w_{i-1}+w_{i-2}\right)+a^{2}\left(\frac{\rho I}{E I} \omega^{2}-\frac{k}{K_{S} G A}+\frac{\rho A \omega^{2}}{K_{S} G A}\right)\left(w_{i+1}-2 w_{i}+w_{i-1}\right)+ \\
a^{4}\left(\frac{k}{4 E I}-\frac{\rho A \omega^{2}}{4 E I}\right)\left(w_{i+1}+2 w_{i}+w_{i-1}\right)+a^{4}\left(-\frac{k \rho I \omega^{2}}{E I K_{S} G A}+\frac{\rho^{2} I A \omega^{4}}{E I K_{S} G A}\right) w_{i}=0 \\
\left(\theta_{i+2}-4 \theta_{i+1}+6 \theta_{i}-4 \theta_{i-1}+\theta_{i-2}\right)+a^{2}\left(\frac{\rho I}{E I} \omega^{2}-\frac{k}{K_{S} G A}+\frac{\rho A \omega^{2}}{K_{S} G A}\right)\left(\theta_{i+1}-2 \theta_{i}+\theta_{i-1}\right)+ \\
a^{4}\left(\frac{k}{4 E I}-\frac{\rho A \omega^{2}}{4 E I}\right)\left(\theta_{i+1}+2 \theta_{i}+\theta_{i-1}\right)+a^{4}\left(-\frac{k \rho I \omega^{2}}{E I K_{S} G A}+\frac{\rho^{2} I A \omega^{4}}{E I K_{S} G A}\right) \theta_{i}=0
\end{gathered}
$$

As was mentioned in the previous section these two equation systems are true for all grains except the two ends. For simply supported boundary conditions as shown in figure 1 and with respect to Eq. (8), the four boundary conditions are formulated as:

$$
\left\{\begin{array}{c}
W_{0}=0 \\
W_{n}=0 \\
C\left(\Theta_{1}-\Theta_{0}\right)+\frac{a}{2} S\left(W_{1}-W_{0}\right)-\frac{a^{2}}{4} S\left(\Theta_{1}+\Theta_{0}\right)-I_{m_{0}} \ddot{\Theta}_{0}=0 \\
C\left(\Theta_{n}-\Theta_{n-1}\right)+\frac{a}{2} S\left(W_{n}-W_{n-1}\right)-\frac{a^{2}}{4} S\left(\Theta_{n}+\Theta_{n-1}\right)+I_{m_{n}} \ddot{\Theta}_{n}=0
\end{array}\right.
$$

The two last equations of Eq. (22) are actually second-newton laws for the boundary grains and could be rewritten in the compact form by

$$
\begin{gathered}
M_{1 / 2}+\frac{a}{2} V_{1 / 2}=I_{m_{0}} \ddot{\Theta}_{0} \\
-M_{n-1 / 2}-\frac{a}{2} V_{n-1 / 2}=I_{m_{n}} \ddot{\Theta}_{n}
\end{gathered}
$$

where

$$
\begin{gathered}
M_{1 / 2}=C\left(\Theta_{1}-\Theta_{0}\right), \quad V_{1 / 2}=\frac{a}{2} S\left(W_{1}-W_{0}-\frac{a}{2}\left(\Theta_{1}+\Theta_{0}\right)\right) \\
M_{n-1 / 2}=C\left(\Theta_{n}-\Theta_{n-1}\right), \quad V_{n-1 / 2}=\frac{a}{2} S\left(W_{n}-W_{n-1}-\frac{a}{2}\left(\Theta_{n}+\Theta_{n-1}\right)\right)
\end{gathered}
$$

On the other hand, Eq. (7) could be applied for the boundary grains by considering two fictitious grains $(i=-1$ and $i=n+1)$ connected to the system with fictitious springs. The equilibrium conditions of the boundary grains could be written by Eq. (25).

$$
\begin{gathered}
M_{1 / 2}-M_{-1 / 2}+\frac{a}{2}\left(V_{1 / 2}-V_{-1 / 2}\right)=\tilde{I}_{m_{0}} \ddot{\Theta}_{0} \\
M_{n-1 / 2}-M_{n+1 / 2}+\frac{a}{2}\left(V_{n-1 / 2}-V_{n+1 / 2}\right)=\tilde{I}_{m_{n}} \ddot{\Theta}_{n}
\end{gathered}
$$

where

$$
\begin{gathered}
M_{-1 / 2}=C\left(\Theta_{0}-\Theta_{-1}\right), \quad V_{-1 / 2}=\frac{a}{2} S\left(W_{0}-W_{-1}-\frac{a}{2}\left(\Theta_{0}+\Theta_{-1}\right)\right) \\
M_{n+1 / 2}=C\left(\Theta_{n+1}-\Theta_{n}\right), \quad V_{n+1 / 2}=\frac{a}{2} S\left(W_{n+1}-W_{n}-\frac{a}{2}\left(\Theta_{n+1}+\Theta_{0}\right)\right)
\end{gathered}
$$

The antisymmetric conditions lead to: 


$$
\begin{array}{cl}
M_{-1 / 2}=-M_{1 / 2}, & V_{-1 / 2}=V_{1 / 2} \\
M_{n+1 / 2}=-M_{n-1 / 2}, & V_{n+1 / 2}=V_{n-1 / 2}
\end{array}
$$

Ones could be concluded that

$$
\begin{array}{cc}
\Theta_{1}=\Theta_{-1}, & W_{1}=-W_{-1} \\
\Theta_{n-1}=\Theta_{n+1}, & W_{n-1}=-W_{n+1}
\end{array}
$$

Using the recent conditions in Eq. (7) for $i=0$ and $i=n$, leads to

$$
\begin{gathered}
C\left(\Theta_{1}-\Theta_{0}\right)+\frac{a}{2} S\left(W_{1}-W_{-1}-\frac{a}{2}\left(\Theta_{1}+\Theta_{0}\right)\right)-\frac{\tilde{I}_{m_{0}}}{2} \ddot{\Theta}_{0}=0 \\
C\left(\Theta_{n-1}-\Theta_{n}\right)+\frac{a}{2} S\left(W_{n+1}-W_{n-1}-\frac{a}{2}\left(\Theta_{n}+\Theta_{n-1}\right)\right)-\frac{\tilde{I}_{m_{n}}}{2} \ddot{\Theta}_{n}=0
\end{gathered}
$$

where $\tilde{I}_{m_{0}}$ and $\tilde{I}_{m_{n}}$ represent the second moment of inertia for the boundary grains with consideration of the fictitious elements $(i=-1$ and $n+1)$.

Comparing the two systems of equations Eq. (22) and Eq. (29) result that the fictitious system behaves the same as the real model with the associated boundary conditions as follows:

$$
\begin{aligned}
& \text { At } i=0: \quad w_{0}=0 ; w_{1}=-w_{-1} \rightarrow \delta_{2} w_{0}=0 \\
& \text { At } i=n: w_{n}=0 ; w_{n-1}=-w_{n+1} \rightarrow \delta_{2} w_{n}=0
\end{aligned}
$$

These boundary conditions have been used also by Hunt et al. [33] for the problem of static bifurcation of granular chains under axial load. The non-dimensional quantities may be introduced

$$
\Omega^{2}=\frac{\omega^{2} \rho A L^{4}}{E I}, \mu_{S}=\frac{E}{K_{S} G}, r=\sqrt{\frac{I}{A}}, r^{*}=\frac{r}{L}, k^{*}=\frac{k L^{4}}{E I}
$$

$\Omega$ is a dimensionless frequency; $\mu_{s}$ is inversely proportional to the shear stiffness; and $r^{*}$ is proportional to the rotatory inertia. The solution of the linear difference equation is thought in the form

$$
w_{i}=B \lambda^{i}
$$

where $B$ is a constant. Therefore the characteristic equation could be obtained by replacing Eq. (32) in Eq. (20) as

$$
\left(\lambda+\frac{1}{\lambda}\right)^{2}+\left(\lambda+\frac{1}{\lambda}\right) \epsilon+\tau=0
$$

where the parameters $\epsilon$ and $\tau$ can be defined as

$$
\begin{gathered}
\epsilon=\left[\frac{r^{* 2} \Omega^{2}}{n^{2}}\left(1+\mu_{s}-\frac{1}{4 r^{* 2} n^{2}}\right)-\frac{r^{* 2} k^{*} \mu_{s}}{n^{2}}+\frac{k^{*}}{4 n^{4}}-4\right], \\
\tau=\left[\frac{r^{* 2} \Omega^{2}}{n^{4}}\left(-\mu_{s} r^{* 2} k^{*}+\mu_{s} r^{* 2} \Omega^{2}\right)+2\left(\frac{k^{*}}{4 n^{4}}-\frac{\Omega^{2}}{4 n^{4}}\right)-2\left(\frac{r^{* 2} \Omega^{2}}{n^{2}}\left(1+\mu_{s}\right)-\frac{r^{* 2} k^{*} \mu_{s}}{n^{2}}\right)+4\right]
\end{gathered}
$$

Solving Eq. (33) leads to the equation obtained by Zhang et al. [34] which could be written 


$$
\lambda+\frac{1}{\lambda}=\frac{-\epsilon \pm \sqrt{\epsilon^{2}-4 \tau}}{2}
$$

Eq. (35) admits four solutions written

$$
\begin{aligned}
& \lambda_{1,2}=\frac{-\epsilon+\sqrt{\epsilon^{2}-4 \tau}}{4} \pm \sqrt{\left(\frac{\epsilon-\sqrt{\epsilon^{2}-4 \tau}}{4}\right)^{2}-1} \\
& \lambda_{3,4}=\frac{-\epsilon-\sqrt{\epsilon^{2}-4 \tau}}{4} \pm j \sqrt{1-\left(\frac{\epsilon+\sqrt{\epsilon^{2}-4 \tau}}{4}\right)^{2}}
\end{aligned}
$$

where $j^{2}=-1$. On the other hand, it is important to notice that according to Eq. (35) the results of $\lambda+\frac{1}{\lambda}$ are in the ranges of $(-\infty,-2]$ or $[2,+\infty)$.

The limited cases when $\lambda+\frac{1}{\lambda}= \pm 2$ would happen for $\lambda= \pm 1$ which refers to the critical frequencies. The critical frequencies of the system are inconsistent condition with Eq. (32) would be obtained by assuming:

$$
\tau= \pm 2 \epsilon-4
$$

Replacing $\tau$ and $\epsilon$ by using Eq. (34), ones could be obtained as:

$$
\begin{gathered}
\left(\frac{r^{* 2} \Omega_{c r}^{2}}{n^{2}}-4\right)\left(\frac{\mu_{s} r^{* 2}}{n^{2}}\left(\Omega_{c r}^{2}-k^{*}\right)-4\right)=0 \\
\frac{1}{n^{4}}\left(\left(\Omega_{c r}^{2}-k^{*}\right)\left(\mu_{s} r^{* 4} \Omega_{c r}^{2}-1\right)\right)=0
\end{gathered}
$$

Therefore, two branches of critical frequencies would be obtained as follows

$$
\begin{gathered}
\Omega_{c r_{1,1}}=\frac{2 n}{r^{*}}, \quad \Omega_{c r_{1,2}}=\sqrt{\frac{4 n^{2}}{\mu_{s} r^{* 2}}+k^{*}} \\
\Omega_{c r_{2,1}}=\sqrt{k^{*}} \quad, \quad \Omega_{c r_{2,2}}=\sqrt{\frac{1}{\mu_{s} r^{* 4}}}
\end{gathered}
$$

The critical frequencies of the first branch depend on the grain number (microstructure parameter), mechanical properties and beam geometry (macrostructure parameters) while the second branch critical frequencies are only defined as a function of the beam mechanical properties and geometry. On the other hand, comparing these critical values with the those of the Timoshenko continuum beam resting on the Winkler foundations (see Wang and Stephens [15]), leads to the equivalency of the second branch critical values (Eq. (42)) to the Timoshenko continuum beam's. For an infinite number of grains, since the first branch critical frequencies $\left(\Omega_{c r_{1,1}}\right.$ and $\Omega_{c r_{1,2}}$ ) leads to infinite values and consequently disappear, so only the second branch would remain. These critical values could be shown as follows

$$
\omega_{c r_{2,1}}=\omega_{\text {cr }_{\text {Timoshenko 1 }}}=\sqrt{\frac{k}{\rho A}} \quad, \quad \omega_{c r_{2,2}}=\omega_{\text {cr }_{\text {Timoshenko } 2}}=\sqrt{\frac{K_{s} G A}{\rho I}}
$$


The behavior of the beam deflection solution would be separated by the critical frequencies into different regimes and depending on the frequencies values, the results would be in a distinct manner.

For a finite number of grains, four regimes would be occurred categorized as follows: when $0<\Omega<\Omega_{c r_{2,2}}$ there are two exponential terms and two traveling waves since $\lambda_{1,2}$ are real and $\lambda_{3,4}$ are imaginary. In this case, the deflection equation form would be obtained from Eq. (54).

When $\Omega_{c r_{2,2}}<\Omega<\Omega_{c r_{1,2}}, \lambda_{1,2,3,4}$ are all imaginary and therefore all terms represent traveling waves and for this case the deflection equation form would be obtained from Eq. (55).

For $\Omega_{c r_{1,2}}<\Omega<\Omega_{c r_{1,1}}$ again there are two exponential terms and two traveling waves since $\lambda_{1,2}$ are imaginary and $\lambda_{3,4}$ are real and the deflection equation form would be obtained from Eq. (56).

Finally, for $\Omega_{c r_{1,1}}<\Omega$, since all parameters of $\lambda_{1,2,3,4}$ are real, thus whole terms represent exponential terms which leads to the deflection equation form of Eq. (57).

For specific value of grain number $\Omega_{c r_{1,1}}$ and $\Omega_{c r_{2,2}}$ would be equal together. This leads to the reduction of the four regimes to three.

$$
n=\frac{1}{2 r^{*} \sqrt{\mu_{s}}}=\frac{L}{2} \sqrt{\frac{K_{s} G A}{E I}}
$$

The results are shown for a case study of 50 grains and the dimensionless parameters of $\mu_{s}=$ $4.28, r^{*}=0.029, k^{*}=15$ in Figure 2. In this example, the values of the critical frequencies are respectively $\Omega_{c r_{1,1}}=3464.1, \Omega_{c r_{1,2}}=1673.7, \Omega_{c r_{2,1}}=3.87$ and $\Omega_{c r_{2,2}}=579.8$.

(a)

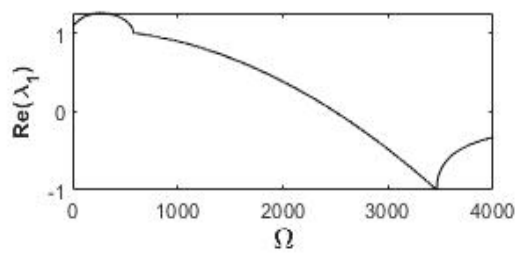

(c)

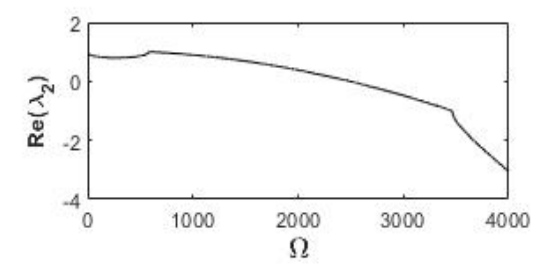

(e)

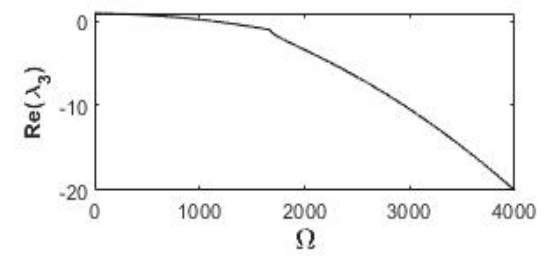

(g) (b)

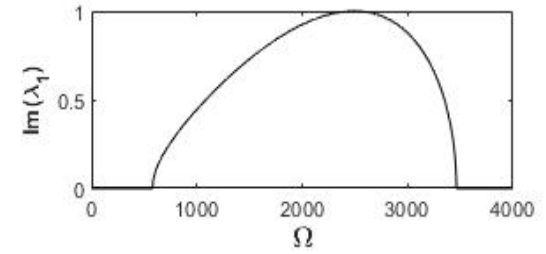

(d)

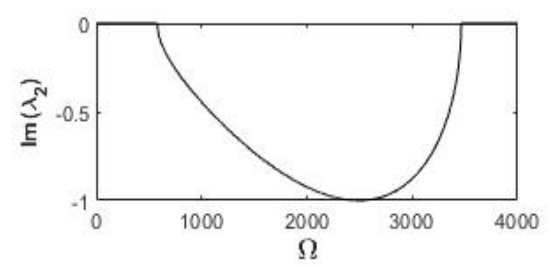

(f)

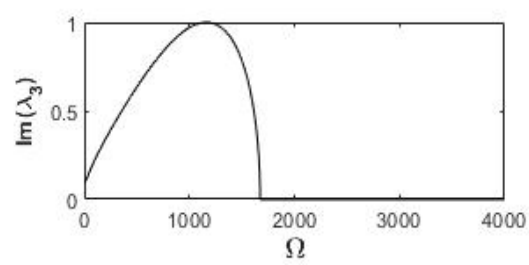

(h) 

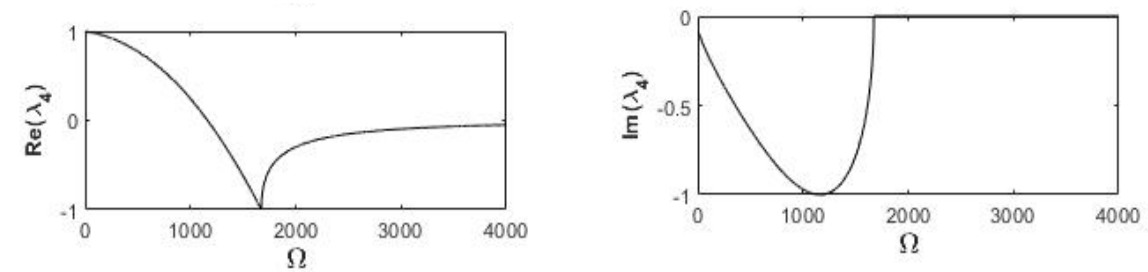

Fig. 2. Schematic behavior of the wave vector regarding the eigenfrequencies for finite grain number ( $\mathrm{n}=50)$. (a), (c), (e) and (g) correspond to the real part and (b), (d), (f) and (h) correspond to the imaginary part of the wave vector.

For an infinite number of grains, since the first two critical values converge to the infinite, so the previous different regimes reduce to two regimes: when $0<\Omega<\Omega_{c r_{2,2}}$ there are two exponential terms and two traveling waves as $\lambda_{1,2}$ are real and $\lambda_{3,4}$ are imaginary and thus the deflection equation form would be obtained from Eq. (54).

When $\Omega_{c r_{2,2}}<\Omega, \lambda_{1,2,3,4}$ are all imaginary and thus all terms represent traveling waves. This case leads to the deflection equation form of Eq. (55). These two regimes correspond to the ones obtained for the continuum beam of Timoshenko resting on Winkler foundations.

(a)

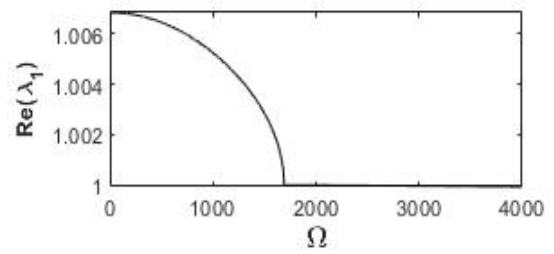

(c)

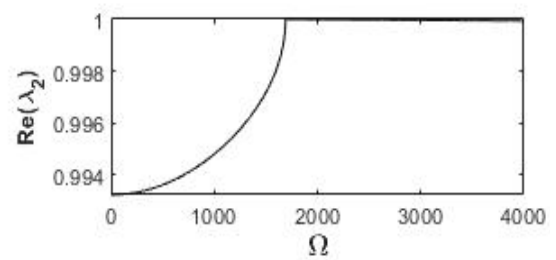

(e)

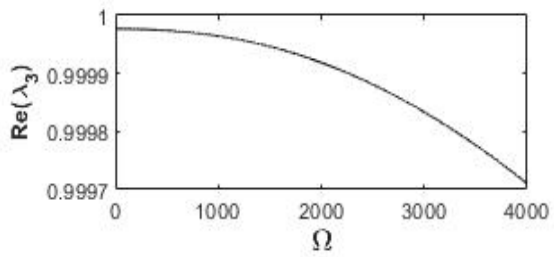

(g)

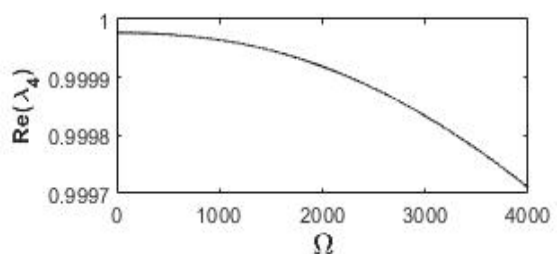

(b)

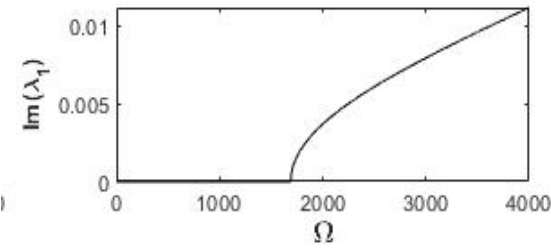

(d)

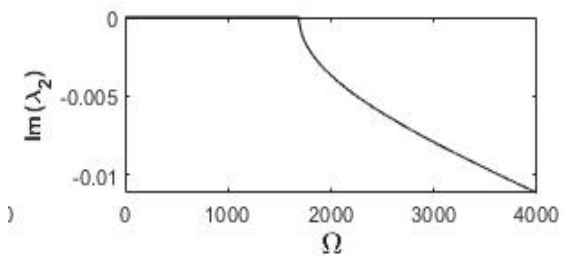

(f)

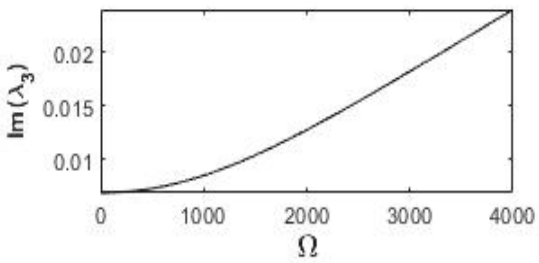

(h)

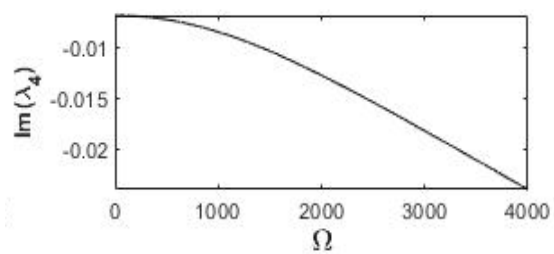

Fig. 3. The effects of the eigenfrequencies on the wave behavior for a general discrete beam contains infinite grain number $(n \rightarrow \infty)$. (a), (c), (e) and (g) correspond to the real part and (b), (d), (f) and (h) correspond to the imaginary part of the wave vector.

Therefore, $\lambda_{1,2}$ can be rewritten for $\Omega<\Omega_{c r_{2,2}}$ and $\Omega_{c r_{1,2}}<\Omega$ (see also Elishakoff and Santoro [32] for a similar presentation applied to the finite difference formulation of Euler-Bernoulli beams) as 


$$
\lambda_{1,2}=\cosh \vartheta \pm \sinh \vartheta
$$

where

$$
\begin{gathered}
\cosh \vartheta=\frac{-\epsilon}{4}+\frac{1}{2} \sqrt{\left(\frac{-\epsilon}{2}\right)^{2}-\tau}=\frac{-\epsilon}{2}-\cos \varphi \\
\cos \varphi=\frac{-\epsilon}{4}-\frac{1}{2} \sqrt{\left(\frac{-\epsilon}{2}\right)^{2}-\tau}
\end{gathered}
$$

while $\lambda_{1,2}$ would be obtained for $\Omega_{c r_{2,2}}<\Omega<\Omega_{c r_{1,2}}$

$$
\lambda_{1,2}=\cos \vartheta \pm j \sin \vartheta
$$

where

$$
\cos \vartheta=\frac{-\epsilon}{4}+\frac{1}{2} \sqrt{\left(\frac{-\epsilon}{2}\right)^{2}-\tau}
$$

On the other hand, $\lambda_{3,4}$ would be defined for $\Omega<\Omega_{c r_{1,1}}$ by

$$
\lambda_{3,4}=\cos \varphi \pm j \sin \varphi
$$

where

$$
\varphi=\arccos \left(\frac{-\epsilon}{4}-\frac{1}{2} \sqrt{\left.\left(\frac{-\epsilon}{2}\right)^{2}-\tau\right)}\right.
$$

And for $\Omega_{c r_{1,1}}<\Omega$

$$
\begin{aligned}
& \lambda_{3,4}=\cosh \varphi \pm \sinh \varphi \\
& \varphi=\operatorname{arccosh}\left(\frac{-\epsilon}{4}-\frac{1}{2} \sqrt{\left(\frac{-\epsilon}{2}\right)^{2}-\tau}\right)
\end{aligned}
$$

In view of Eq. (46) and (47), there are three possible general solutions for $w_{i}$ depending on the critical values of the frequencies which may be represented as

$$
\begin{gathered}
w_{i}=A_{1} \cos i \varphi+A_{2} \sin i \varphi+A_{3} \cosh i \vartheta+A_{4} \sinh i \vartheta\left(\Omega<\Omega_{c r_{2,2}}\right) \\
w_{i}=B_{1} \cos i \varphi+B_{2} \sin i \varphi+B_{3} \cos i \vartheta+B_{4} \sin i \vartheta\left(\Omega_{c r_{2,2}}<\Omega<\Omega_{c r_{1,2}}\right) \\
w_{i}=C_{1} \cosh i \varphi+C_{2} \sinh i \varphi+C_{3} \cos i \vartheta+C_{4} \sin i \vartheta\left(\Omega_{c r_{1,2}}<\Omega<\Omega_{c r_{1,1}}\right) \\
w_{i}=D_{1} \cosh i \varphi+D_{2} \sinh i \varphi+D_{3} \cosh i \vartheta+D_{4} \sinh i \vartheta\left(\Omega_{c r_{1,1}}<\Omega\right)
\end{gathered}
$$

For the simply supported discrete system by substituting Eq. (30) in Eq. (54), (55), (56) and (57) the boundary conditions could be defined in matrix form, respectively 
$\left[\begin{array}{cccc}1 & 0 & 1 & 0 \\ \cos (n \varphi) & \sin (n \varphi) & \cosh (n \vartheta) & \sinh (n \vartheta) \\ 2 \cos \varphi & 0 & 2 \cosh \vartheta & 0 \\ 2 \cos \varphi \cos (n \varphi) & 2 \cos \varphi \sin (n \varphi) & 2 \cosh \vartheta \cosh (n \vartheta) & 2 \cosh \vartheta \sinh (n \vartheta)\end{array}\right]\left[\begin{array}{l}A_{1} \\ A_{2} \\ A_{3} \\ A_{4}\end{array}\right]=0$

$\left[\begin{array}{cccc}1 & 0 & 1 & 0 \\ \cos (n \varphi) & \sin (n \varphi) & \cos (n \vartheta) & \sin (n \vartheta) \\ 2 \cos \varphi & 0 & 2 \cos \vartheta & 0 \\ 2 \cos \varphi \cos (n \varphi) & 2 \cos \varphi \sin (n \varphi) & 2 \cos \vartheta \cos (n \vartheta) & 2 \cos \vartheta \sin (n \vartheta)\end{array}\right]\left[\begin{array}{l}B_{1} \\ B_{2} \\ B_{3} \\ B_{4}\end{array}\right]=0$

$\left[\begin{array}{cccc}1 & 0 & 1 & 0 \\ \cosh (n \varphi) & \sinh (n \varphi) & \cos (n \vartheta) & \sin (n \vartheta) \\ 2 \cosh \varphi & 0 & 2 \cos \vartheta & 0 \\ 2 \cosh \varphi \cos h(n \varphi) & 2 \cosh \varphi \sinh (n \varphi) & 2 \cos \vartheta \cos (n \vartheta) & 2 \cos \vartheta \sinh (n \vartheta)\end{array}\right]\left[\begin{array}{l}C_{1} \\ C_{2} \\ C_{3} \\ C_{4}\end{array}\right]=0$

$$
\left.\begin{array}{rccc}
1 & 0 & 1 & 0 \\
\cosh (n \varphi) & \sinh (n \varphi) & \cosh (n \vartheta) & \sinh (n \vartheta) \\
2 \cosh \varphi & 0 & 2 \cosh \vartheta & 0 \\
2 \cosh \varphi \cosh (n \varphi) & 2 \cosh \varphi \sinh (n \varphi) & 2 \cosh \vartheta \cosh (n \vartheta) & 2 \cosh \vartheta \sinh (n \vartheta)
\end{array}\right]\left[\begin{array}{l}
D_{1} \\
D_{2} \\
D_{3} \\
D_{4}
\end{array}\right]
$$

Setting the determinant of the homogeneous coefficient matrix of Eq. (58), (59), (60) and (61) to zero would be simplified

$$
\begin{gathered}
4 \sin (n \varphi) \sinh (n \vartheta)(\cos \varphi-\cosh \vartheta)^{2}=0 \\
4 \sin (n \varphi) \sin (n \vartheta)(\cos \varphi-\cos \vartheta)^{2}=0 \\
4 \sinh (n \varphi) \sin (n \vartheta)(\cosh \varphi-\cos \vartheta)^{2}=0 \\
4 \sinh (n \varphi) \sinh (n \vartheta)(\cosh \varphi-\cosh \vartheta)^{2}=0
\end{gathered}
$$

It is found from Eq. (62), (63) and (64) that $\sin (n \varphi)=0$ and or $\sin (n \vartheta)=0$. Thus, the natural vibration modes are obtained from the trigonometric shape function $w_{i}=B \sin (i \varphi)$ and or $w_{i}=B \sin (i \vartheta)$ which lead to the fundamental natural vibration frequency, which are associated with the non-trivial condition:

$$
\text { For } \mathrm{i}=\mathrm{n}, w_{i}=0 \Rightarrow \sin (n \Lambda)=0 \Rightarrow \Lambda=\frac{p \pi}{n}, \Lambda=\varphi, \vartheta
$$

On the other hand, one would be obtained from Eq. (65) that $\varphi=\vartheta$ which leads to

$$
\frac{-\epsilon+\sqrt{\epsilon^{2}-4 \tau}}{4}=\frac{-\epsilon-\sqrt{\epsilon^{2}-4 \tau}}{4} \Rightarrow \epsilon^{2}-4 \tau=0
$$

The frequencies could be obtained from Eq. (67) as follows

$$
\Omega=\sqrt{\frac{32 n^{4}-k^{*}+4 k^{*} r^{*} n^{2}\left(1+2 \mu_{s}\left(1+2 r^{* 2} n^{2}\left(1-\mu_{s}\right)\right)\right) \pm A^{*}}{B^{*}}}
$$


Where $A^{*}$ and $B^{*}$ are defined by

$$
\begin{gathered}
A^{*}=16 n^{3} \sqrt{k^{*}\left(k^{*} \mu_{s} r^{* 6}-16 \mu_{s}^{3} r^{* 6} n^{4}+32 \mu_{s}^{2} r^{* 6} n^{4}+8 \mu_{s}^{2} r^{* 4} n^{2}-16 \mu_{s} r^{* 6} n^{4}+4 \mu_{s} r^{* 4} n^{2}-\mu_{s} r^{2}+4 r^{* 4} n^{2}-r^{* 2}\right)+4 n^{2}} \\
B^{*}=-16 \mu_{s}{ }^{2} r^{* 4} n^{4}+32 \mu_{s} r^{* 4} n^{4}+8 \mu_{s} r^{* 2} n^{2}-16 r^{* 4} n^{4}+8 r^{* 2} n^{2}-1
\end{gathered}
$$

For a simplified case by neglecting the elastic foundation, one could be obtained for the dynamic response as

$$
\Omega=\frac{8 n^{2} \sqrt{\left(4 r^{*} n+\left(4 r^{* 2} n^{2}\left(\mu_{s}-1\right)-1\right)\right)\left(4 r^{*} n-\left(4 r^{* 2} n^{2}\left(\mu_{s}-1\right)-1\right)\right)}}{B^{*}}
$$

the exact resolution of the dynamic analysis of the granular system that would be studied here is only true for the frequencies lower than $\Omega_{c r_{1,2}}$. Since $\Omega_{c r_{1,2}}$ is a function of grain number, the results could be compared well for an infinite number of grains with the ones of the Timoshenko continuum beam.

Therefore, regarding to Eq. (66) the deflection and rotation angle of each grain could be obtained by the following equation while $\Omega<\Omega_{c r_{1,2}}$

$$
w_{i}=\mathrm{B} \sin \left(\frac{i p \pi}{n}\right)
$$

where $p$ is the mode number or natural number $\left(1 \leq p<n\right.$ for $w_{i}$ and $0 \leq p \leq n$ for $\left.\theta_{i}\right)$ and $i$ is the grain number $(0 \leq i \leq n)$.

By substituting Eq. (66) in Eq. (47), one could be written

$$
\begin{gathered}
\cos \left(\frac{p \pi}{n}\right)=\frac{-\epsilon}{4}-\frac{1}{2} \sqrt{\left(\frac{-\epsilon}{2}\right)^{2}-\tau} \\
2 \epsilon \cos \left(\frac{p \pi}{n}\right)+\tau+4\left(\cos \left(\frac{p \pi}{n}\right)\right)^{2}=0
\end{gathered}
$$

which is a quartic equation. Using non-dimensional eigenfrequency parameters

$$
\begin{gathered}
{\left[\frac{\mu_{s} *^{* 4}}{n^{4}}\right] \Omega^{4}+\left[\frac{2 r^{* 2}}{n^{2}}\left(1+\mu_{s}-\frac{1}{4 r^{* 2} n^{2}}\right) \cos \left(\frac{p \pi}{n}\right)-\frac{\mu_{s} r^{* 4} k^{*}}{n^{4}}-\frac{1}{2 n^{4}}-\frac{2 r^{* 2}}{n^{2}}\left(1+\mu_{s}\right)\right] \Omega^{2}+} \\
{\left[2\left(-\frac{r^{* 2} k^{*} \mu_{S}}{n^{2}}+\frac{k^{*}}{4 n^{4}}-4\right) \cos \left(\frac{p \pi}{n}\right)+\frac{k^{*}}{2 n^{4}}+\frac{2 r^{* 2} k^{*} \mu_{s}}{n^{2}}+4+4\left(\cos \left(\frac{p \pi}{n}\right)\right)^{2}\right]=0}
\end{gathered}
$$

Neglecting the terms of Winkler elastic foundation ( $\left.k^{*}=0\right)$, Eq. (75) leads to

$$
\left[\frac{\mu_{s} *^{* 4}}{n^{4}}\right] \Omega^{4}-\left[\frac{4 *^{* 2}}{n^{2}}\left(1+\mu_{s}\right) \sin ^{2}\left(\frac{p \pi}{2 n}\right)+\frac{1}{n^{4}} \cos ^{2}\left(\frac{p \pi}{2 n}\right)\right] \Omega^{2}+\left[16 \sin ^{4}\left(\frac{p \pi}{2 n}\right)\right]=0
$$

The last equation is different from the quartic equation of Duan et al. [24] that had been obtained as follows:

$$
\left[\frac{\mu_{s} r^{* 4}}{n^{4}}\right] \Omega^{4}-\left[\frac{4 r^{* 2}}{n^{2}}\left(1+\mu_{s}\right) \sin ^{2}\left(\frac{p \pi}{2 n}\right)+\frac{1}{n^{4}}\right] \Omega^{2}+\left[16 \sin ^{4}\left(\frac{p \pi}{2 n}\right)\right]=0
$$

Going back to Eq. (75), it could be written in the compact form 


$$
\Omega^{4}-B \Omega^{2}+C=0
$$

in which the coefficients of $B$ and $C$ are defined

$$
\begin{aligned}
& B=-\frac{2 n^{2}}{\mu_{s} r^{* 2}}\left(1+\mu_{s}-\frac{1}{4 r^{* 2} n^{2}}\right) \cos \left(\frac{p \pi}{n}\right)+k^{*}+\frac{1}{2 \mu_{s} r^{* 4}}+\frac{2 n^{2}}{\mu_{s} r^{* 2}}\left(1+\mu_{s}\right), \\
& C=\frac{2 n^{4}}{\mu_{s} r^{* 4}}\left(-\frac{r^{* 2} k^{*} \mu_{s}}{n^{2}}+\frac{k^{*}}{4 n^{4}}-4\right) \cos \left(\frac{p \pi}{n}\right)+\frac{k^{*}}{2 \mu_{s} r^{* 4}}+\frac{2 n^{2} k^{*}}{r^{* 2}}+\frac{4 n^{4}}{\mu_{s} r^{* 4}}\left(1+\cos \left(\frac{p \pi}{n}\right)\right)^{2}
\end{aligned}
$$

Eq. (78) has two real positive roots

$$
\Omega=\sqrt{\frac{B \pm \sqrt{B^{2}-4 C}}{2}}
$$

Eq. (80) shows that for a given mode number (p), there are two valid positive roots which refer to two branches of the eigenfrequency spectrum, in distinction of the one obtained for the Euler-Bernoulli beam associated with a single positive root. The same phenomenon for the continuum Bresse-Timoshenko beam has been already investigated by Traill-Nash and Collar [35] and Manevich [16].

The natural frequencies of the granular chain represented in Figure 1 could be presented in a single form

$$
\omega=\frac{\Omega}{L^{2}} \sqrt{\frac{E I}{\rho A}}
$$

Substituting Eq. (80) in Eq. (81) give the exact eigenfrequencies of the granular beam as a function of grain number $(n)$ and for whatever mode numbers $(p)$.

The recent natural frequency was obtained by the assumption of $\Omega<\Omega_{c r_{1,2}}$ which means that it needs to be able to support both the low and high frequencies. Therefore, the maximum value of $\gamma$ must be less than $\Omega_{c r_{1,2}}$. Here the validity of this hypothesis is checked by analyzing the behavior of Eq. (81). Since $\gamma$ is an ascending function of mode number ( $p$ ) and knowing the mode number values cannot exceed the grain number, thus the maximum value of $\gamma$ could be obtained for $p=n$ which leads to

$$
\gamma_{\max }=\sqrt{\frac{k^{*}}{2}+\frac{2 n^{2}}{\mu_{s} r^{* 2}}\left(1+\mu_{s}\right) \pm \sqrt{\left(\frac{k^{*}}{2}+\frac{2 n^{2}}{\mu_{s} r^{* 2}}\left(1+\mu_{s}\right)\right)^{2}-\frac{n^{4}}{\mu_{s} r^{* 4}}\left(+\frac{4 r^{* 2} k^{*} \mu_{s}}{n^{2}}+16\right)}}
$$

According to the definition of $\Omega_{c r_{1,2}}$ Eq. (82) could be rewritten in the short form as follows

$$
\gamma_{\text {max }}=\sqrt{\frac{\Omega_{c r_{1,2}}{ }^{2}}{2}+\frac{2 n^{2}}{r^{* 2}} \pm \sqrt{\left(\frac{\Omega_{c r_{1,2}}^{2}}{2}+\frac{2 n^{2}}{r^{* 2}}\right)^{2}-\frac{4 n^{2} \Omega_{c r_{1,2}}{ }^{2}}{r^{* 2}}}}
$$

Simplifying Eq. (83), leads to the two max frequency values (Eq. (84)) each refers to the one branch. Therefore, Eq. (81) could be verified well for the range of high-frequency values, the natural frequencies of the discrete system do not exceed their critical values and thus the general solution form of the beam deflection remains in the harmonic and trigonometric manner.

$$
\gamma_{\max , 1}=\Omega_{c r_{1,1}}, \quad \gamma_{\max , 2}=\Omega_{c r_{1,2}}
$$

By considering low mode number $(p<<n)$ and for the continuum case when $n \rightarrow \infty$, the assumption of $\cos \left(\frac{p \pi}{n}\right) \sim 1-\frac{1}{2}\left(\frac{p \pi}{n}\right)^{2}$ could be applied to Eq. (75). This leads to 


$$
\begin{gathered}
{\left[\frac{\mu_{s} r^{*}}{n^{4}}\right] \Omega^{4}+\left[\frac{2 r^{* 2}}{n^{2}}\left(1+\mu_{s}-\frac{1}{4 r^{* 2} n^{2}}\right)\left(1-\frac{1}{2}\left(\frac{p \pi}{n}\right)^{2}\right)-\frac{\mu_{s} r^{* 4} k^{*}}{n^{4}}-\frac{1}{2 n^{4}}-\frac{2 r^{* 2}}{n^{2}}\left(1+\mu_{s}\right)\right] \Omega^{2}+} \\
{\left[2\left(-\frac{r^{* 2} k^{*} \mu_{s}}{n^{2}}+\frac{k^{*}}{4 n^{4}}-4\right)\left(1-\frac{1}{2}\left(\frac{p \pi}{n}\right)^{2}\right)+\frac{k^{*}}{2 n^{4}}+\frac{2 r^{* 2} k^{*} \mu_{s}}{n^{2}}+4+4\left(1-\left(\frac{p \pi}{n}\right)^{2}+\frac{1}{4}\left(\frac{p \pi}{n}\right)^{4}\right)\right]=0}
\end{gathered}
$$

Eq. (85) can be simplified

$$
\Omega^{4}-\left[\frac{p^{2} \pi^{2}}{\mu_{s} r^{* 2}}\left(1+\mu_{S}\right)+k^{*}+\frac{1}{\mu_{s} r^{* 4}}\right] \Omega^{2}+\left[\left(\frac{k^{*} p^{2} \pi^{2}}{r^{* 2}}+\frac{p^{4} \pi^{4}}{\mu_{S} r^{* 4}}\right)+\frac{k^{*}}{\mu_{S} r^{* 4}}\right]=0
$$

and in the compact form

$$
\Omega^{4}-B \Omega^{2}+C=0
$$

where the two coefficients of $\mathrm{B}$ and $\mathrm{C}$ are defined as:

$$
B=\frac{p^{2} \pi^{2}}{\mu_{s} r^{* 2}}\left(1+\mu_{S}\right)+\frac{1}{\mu_{s} r^{* 2}}\left(\mu_{S} r^{* 2} k^{*}+\frac{1}{r^{* 2}}\right), \quad C=\frac{p^{4} \pi^{4}}{\mu_{S} r^{* 4}}+\frac{k^{*} p^{2} \pi^{2}}{r^{* 2}}+\frac{k^{*}}{\mu_{S} r^{* 4}},
$$

Solving the quartic equation of (87) leads to the eigenfrequency values of the continuous beam that would be obtained again by Eq. (81) and with $\gamma$ expressed as follows:

$$
\gamma=\sqrt{\frac{p^{2} \pi^{2}}{2 \mu_{s} r^{* 2}}\left(1+\mu_{s}\right)+\frac{k^{*}}{2}+\frac{1}{2 \mu_{s} r^{* 4}} \pm \sqrt{\left(\frac{p^{2} \pi^{2}}{2 \mu_{s} r^{* 2}}\left(1+\mu_{s}\right)+\frac{k^{*}}{2}+\frac{1}{2 \mu_{s} r^{* 4}}\right)^{2}-\left(\frac{k^{*}}{\mu_{s} r^{* 4}}+\frac{k^{*} p^{2} \pi^{2}}{r^{* 2}}+\frac{p^{4} \pi^{4}}{\mu_{s} r^{* 4}}\right)}}
$$

These results agree with the ones obtained by Wang and Stephens [15], Cheng and Pantelides [28] and Manevich [16]. Also with the negligence of the Winkler elastic foundation $\left(k^{*}=0\right)$, it could be compared well to Timoshenko [10, 11].

\subsection{Continuum Solution}

In the limit case for the continuum beam, the fourth-order differential equation including the Winkler elastic foundation could be considered in dimensionless form

$$
\frac{d^{4} \bar{w}}{d \bar{x}^{4}}+\left[r^{* 2} \Omega^{2}\left(1+\mu_{s}\right)-r^{* 2} k^{*} \mu_{s}\right] \frac{d^{2} \bar{w}}{d \bar{x}^{2}}-\left[r^{* 2} \Omega^{2}\left(\mu_{s} r^{* 2} k^{*}+\frac{1}{r^{* 2}}-\mu_{s} r^{* 2} \Omega^{2}\right)-k^{*}\right] \bar{w}=0
$$

Eq. (90) is obtained by Wang and Stephens [15] and the non-dimensional parameters can be introduced

$$
\bar{x}=\frac{x}{L}, \bar{w}=\frac{w}{L}, \frac{d^{2} \bar{w}}{d X^{2}}=L \frac{d^{2} w}{d x^{2}}, \frac{d^{4} \bar{w}}{d X^{4}}=L^{3} \frac{d^{4} w}{d x^{4}}
$$

For simply supported beam, the solution of Eq. (90) can be proposed by

$$
\bar{w}(\bar{x})=\sin (p \pi \bar{x})
$$

Substituting Eq. (92) in Eq. (90) leads to the following quartic frequency equation.

$$
\left[\mu_{S} r^{* 4}\right] \Omega^{4}-\left[r^{* 2}\left(\mu_{S} r^{* 2} k^{*}+\frac{1}{r^{*}}\right)+r^{* 2} p^{2} \pi^{2}\left(1+\mu_{S}\right)\right] \Omega^{2}+\left[r^{* 2} k^{*} \mu_{S} p^{2} \pi^{2}+k^{*}+p^{4} \pi^{4}\right]=0
$$

which can be considered in the compact form

$$
\Omega^{4}-B \Omega^{2}+C=0
$$

The two coefficients of $B$ and $C$ are defined as: 


$$
B=\frac{p^{2} \pi^{2}}{\mu_{s} r^{* 2}}\left(1+\mu_{s}\right)+\frac{1}{\mu_{s} r^{* 2}}\left(\mu_{S} r^{* 2} k^{*}+\frac{1}{r^{* 2}}\right), \quad C=\frac{p^{4} \pi^{4}}{\mu_{S} r^{* 4}}+\frac{k^{*} p^{2} \pi^{2}}{r^{* 2}}+\frac{k^{*}}{\mu_{s} r^{* 4}}
$$

So, the natural frequencies of the continuous beam could be obtained from the quartic equation of Eq. (94). The results are in the same form as Eq. (81) with substitution of Eq. (89) and can be compared well to Wang and Stephens [15], Cheng and Pantelides [28] and Manevich [16]. If the elastic Winkler foundation is neglected $\left(k^{*}=0\right)$ the eigenfrequency values will be similar to the ones obtained by Timoshenko $[10,11]$.

The sensitivity analysis is performed for the granular chain by assuming the following set of dimensionless parameters for four grain number values $(n=5 ; n=20 ; n=35 ; n=50)$

$$
\mu_{s}=4.28 \text { and } k^{*} \in\{1.875,480,4502,18750\}
$$

In Figure 4, the frequency results obtained by the exact solution of the discrete lattice model have been compared with those of Duan et al. [24]. In this asymptotic analysis, the length of the beam considered constant for instance and by increasing the number of grains subsequently reducing the grain diameter (a) the natural frequencies of the system are obtained. Since the local continuum solution of the problem (mentioned in Eq. (95)) is independent of the grain number, the results do not change by varying the grain number. Each model leads to two branches of frequency.

Regarding to the first branch (lower frequencies), for each typical values of the grain number, the results of two discrete model, diverge from each other and also from the continuum ones by increasing the mode number, starting from two different values of mode number. While for the second branch these two results are close to each other (Figure 5).

(a)

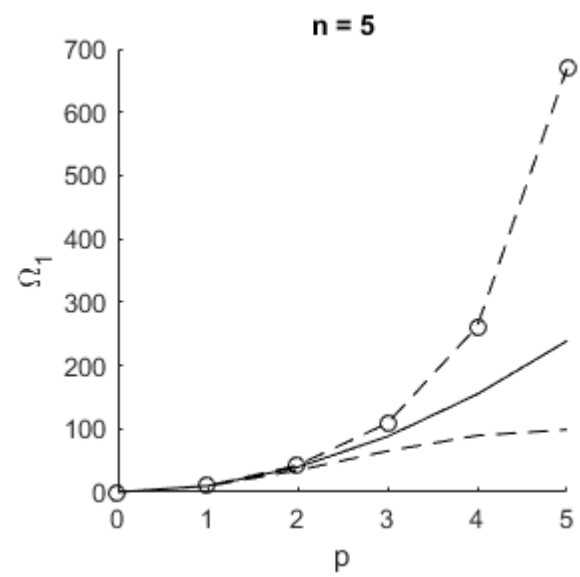

(c) (b)

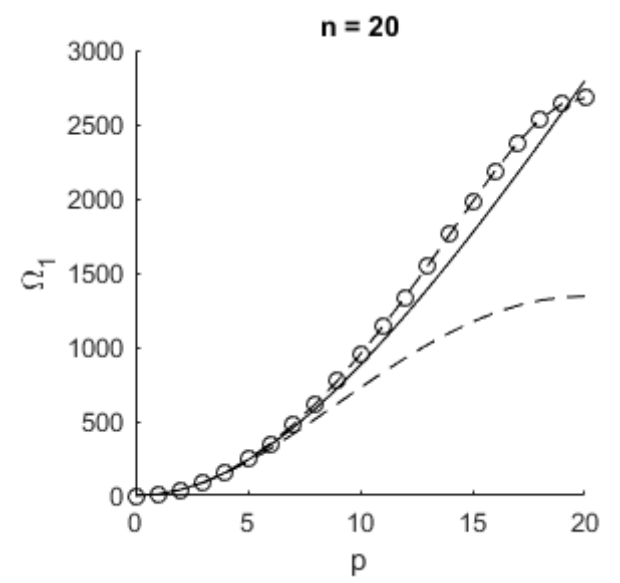

(d) 

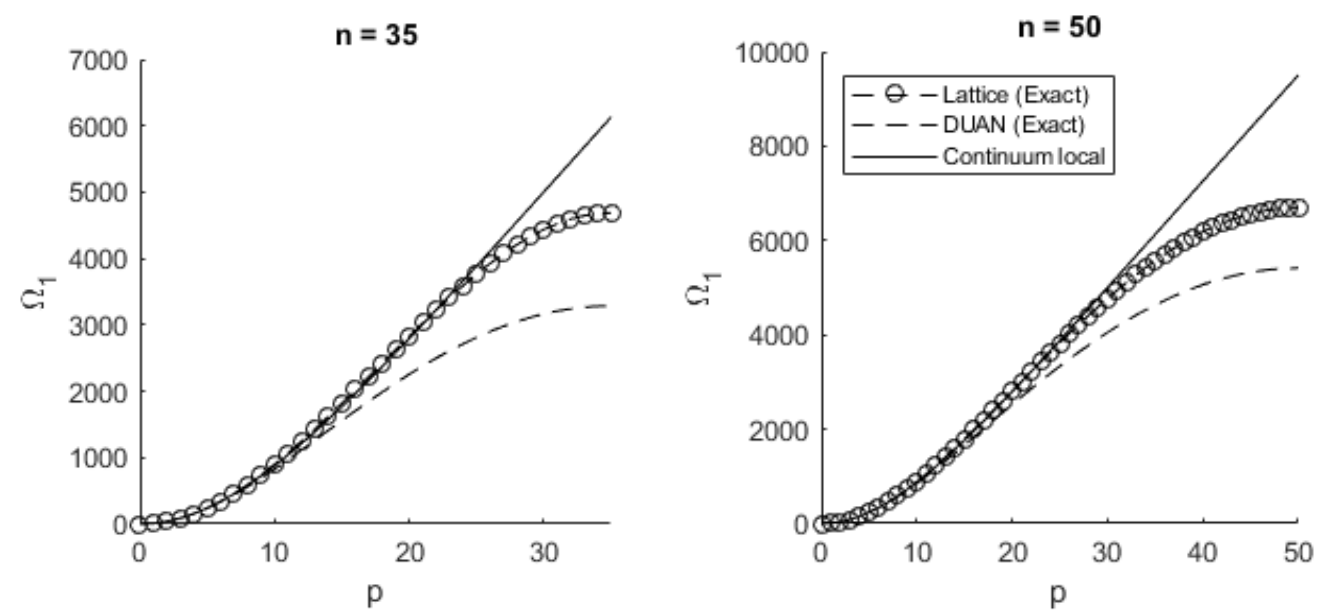

Fig. 4. Comparison of the first branch natural frequencies for the discrete exact, Duan et al. [24] and continuum solutions with respect to the mode number (p) and grain number: (a) $n=5$, (b) $n=20$, (c) $n=35$ and (d) $n=50$ for $\mu_{s}=4.28, r^{*}=0.007$ and $k^{*}=0$.

The results for the second branches of eigenfrequencies have been shown in Figure 5, both for the equivalent continuum beam and the two discrete ones with respect to the mode number (p) and four grain number values $(n=5 ; n=20 ; n=35 ; n=50)$. It can be concluded the exact solution of the discrete model always predicts lower frequencies than the continuum one. As it is expected, by increasing the ratio of $n / p$, the results of the two discrete models converge to the continuous ones. The coincidence of the results happens for the second branch when the ratio of $n / p$ is typically higher than the approximate value of 3 , while this approximate limit value is typically 2 for the first branch.

Furthermore, for the first branch or lower frequencies, the results of the discrete model developed in this paper are closer to the continuum ones in compare with the ones obtained by Duan et al. [24], for a typical value of the mode number. While this conclusion is opposite for the second branch which means for small values of the grain number Duan et al. [24] predict the dynamic response closer to the continuum ones.

(a)

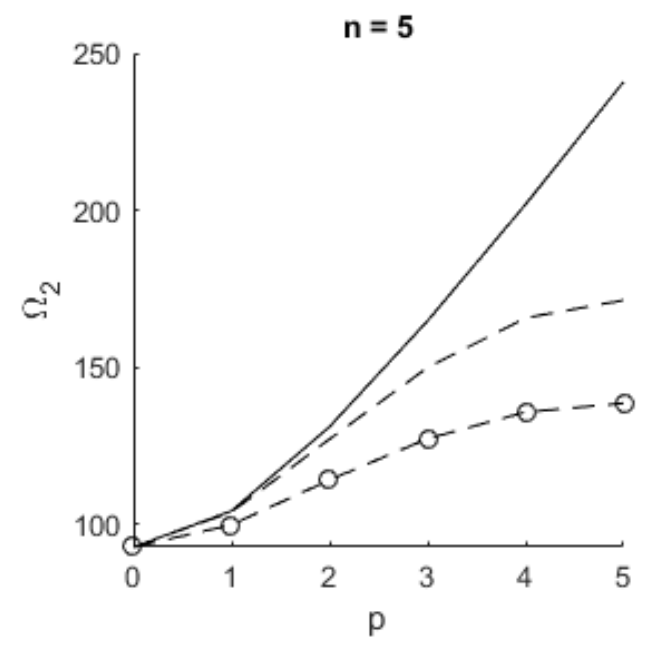

(c) (b)

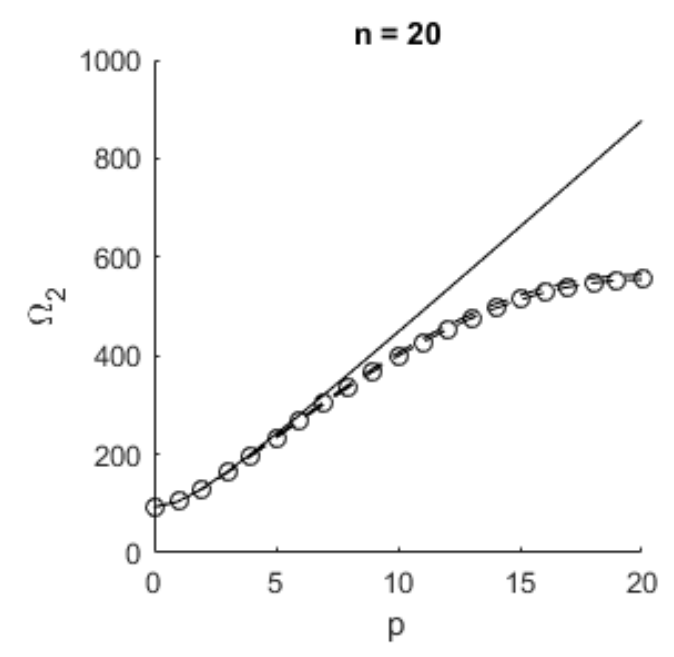

(d) 

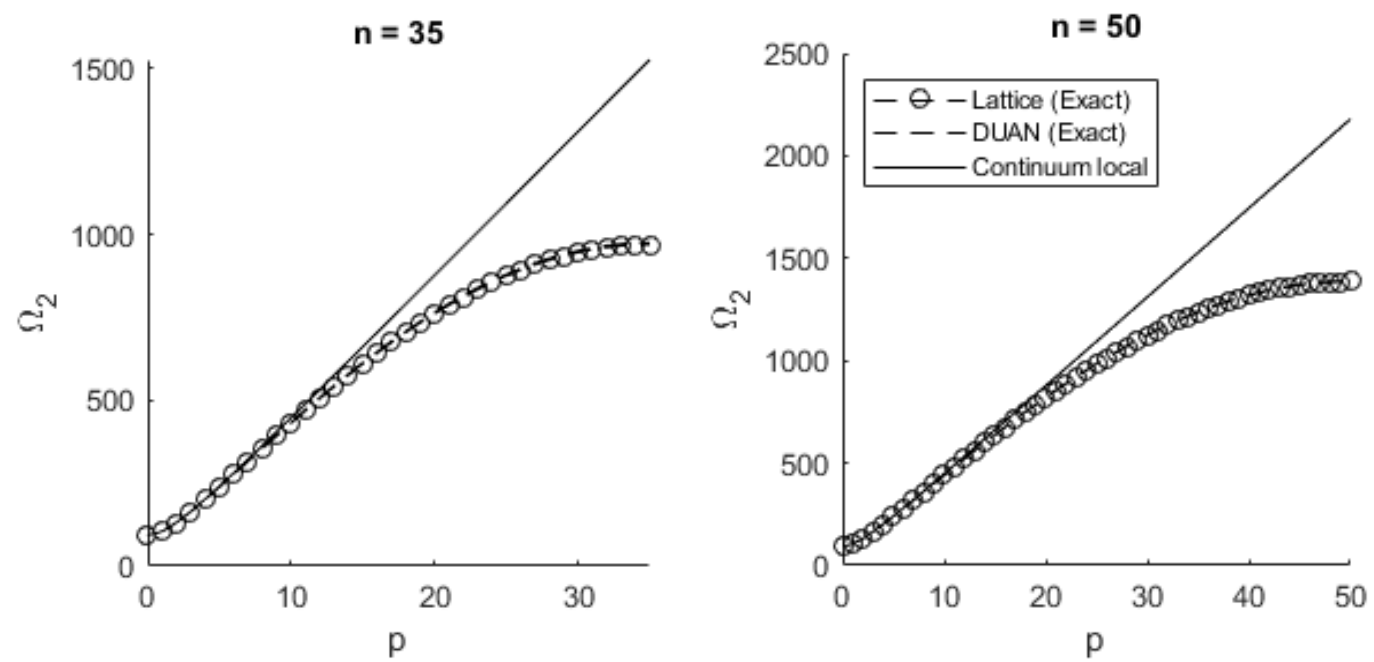

Fig. 5. Comparison of the second branch natural frequencies for the discrete exact, Duan et al. [24] and continuum solutions with respect to the mode number (p) and grain number: (a) $n=5$, (b) $n=20$, (c) $n=35$ and (d) $n=50$ for $\mu_{s}=4.28, r^{*}=0.07$ and $k^{*}=0$.

Here, for a constant grain number and various geometric dimensionless parameters $\left(r^{*}\right)$, the results have been compared and shown for the two branches respectively in Figure. 6 and Figure. 7. Increasing the values of the length ratio, the results obtained by discrete exact solution and Duan et al. [24] converge each other, for both the first and second branches. For the first branch, it can be understood that generally the behavior of the exact discrete solution is closer to the continuum one in compare with Duan et al. [24], for low values of the mode number (p). On the other hand, for the second branch or higher frequencies, the behavior of the results obtained by the exact model introduced in this paper is more sensitive to the length ratio.

(a)

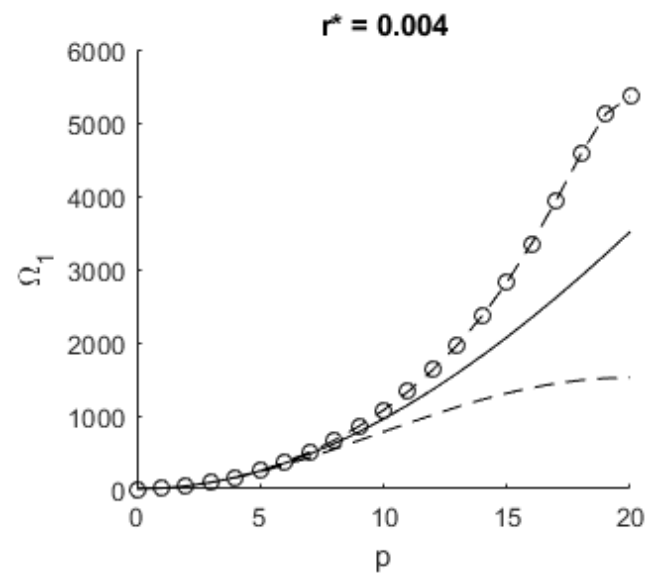

(c) (b)

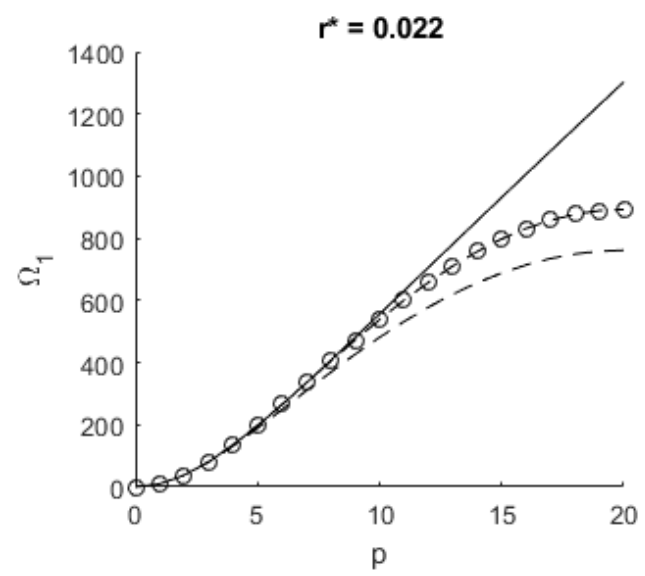

(d) 

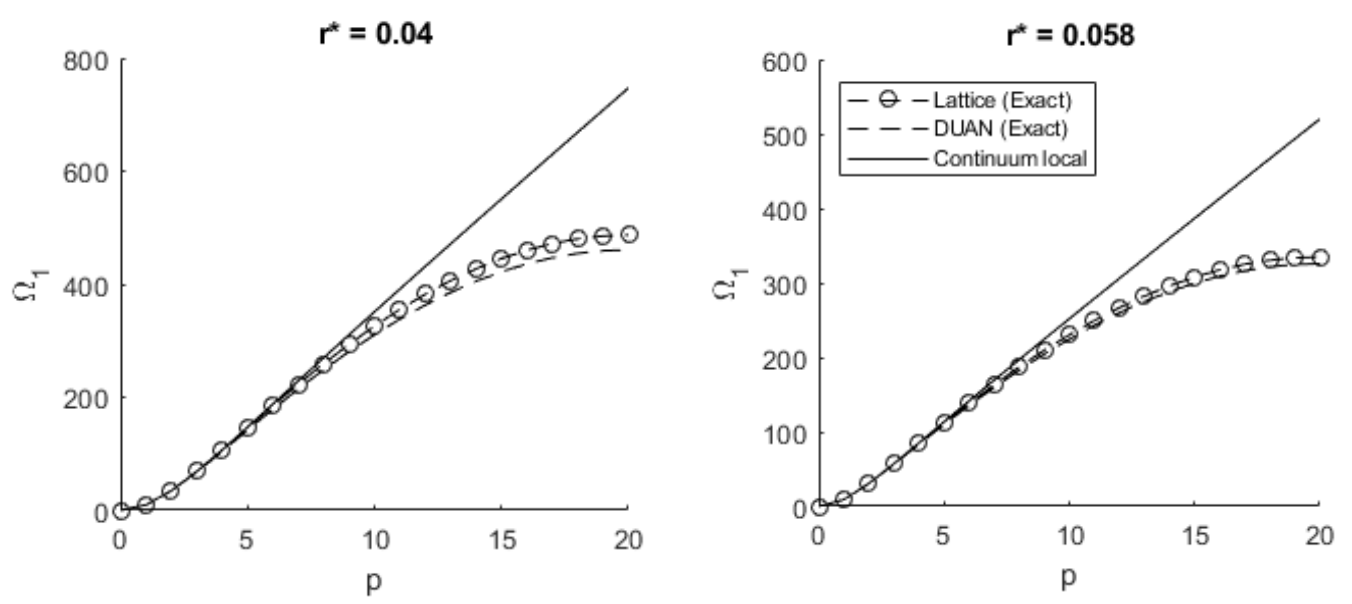

Fig. 6. Comparison of the first branch natural frequencies for the discrete exact, Duan et al. [24] and continuum solutions with respect to the mode number (p) and grain number: (a) $r^{*}=0.004$, (b) $r^{*}=0.022$, (c) $r^{*}=0.04$ and (d) $r^{*}=0.058$ for $n=20, \mu_{s}=4.28$ and $k^{*}=0$.

(a)

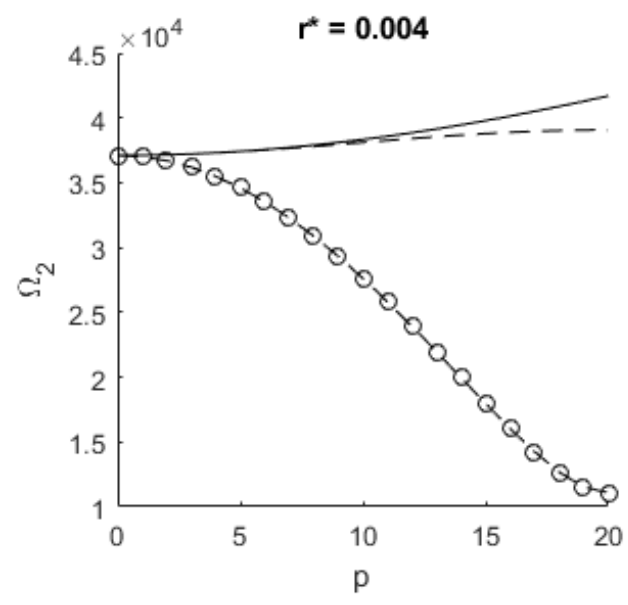

(c)

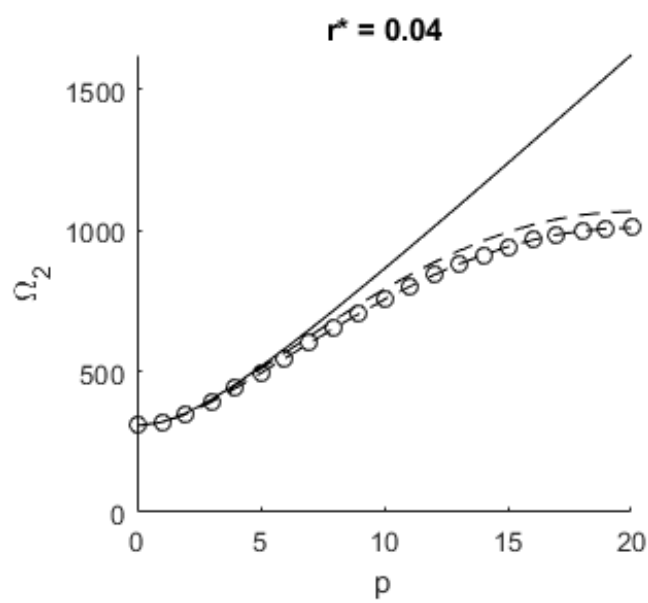

(b)

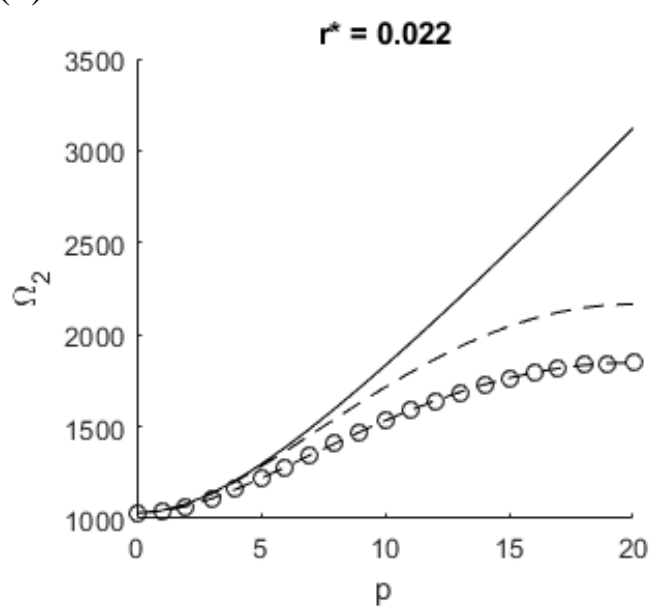

(d)

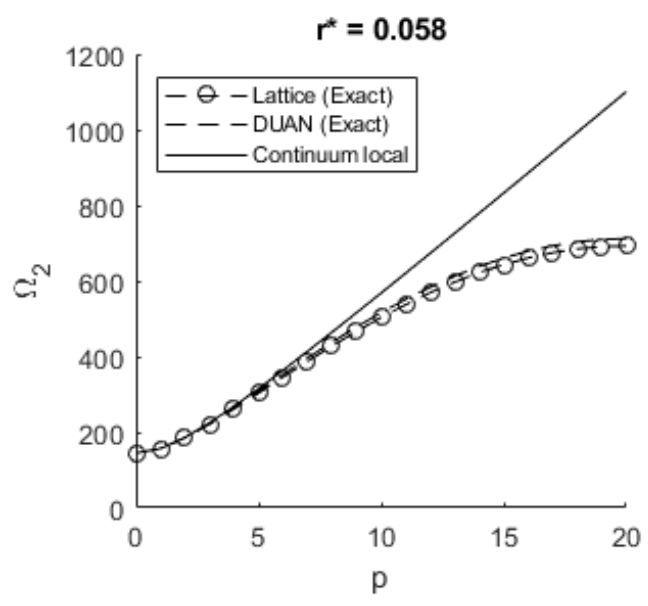

Fig. 7. Comparison of the second branch natural frequencies for the discrete exact, Duan et al. [24] and continuum solutions with respect to the mode number (p) and grain number: (a) $r^{*}=0.004$, (b) $r^{*}=0.022$, (c) $r^{*}=0.04$ and (d) $\mathrm{r}^{*}=0.058$ for $\mathrm{n}=20, \mu_{\mathrm{s}}=4.28$ and $\mathrm{k}^{*}=0$.

In Figure. 8, the effect of length ratio (beam thickness/beam length) regarding to the grain number has been studied for two typical mode number $(\mathrm{p}=1$ and $\mathrm{p}=10)$. The minimum values of the required grain number $\left(n^{*}\right)$ have been also determined and reported when the difference between the discrete and continuum results start to be smaller than $1 \%$. It can be concluded 
generally that in order to achieve the continuum results from discrete solution, whether the length ratio decrease or the mode number increase, the grain number value needs to increase.

(a)

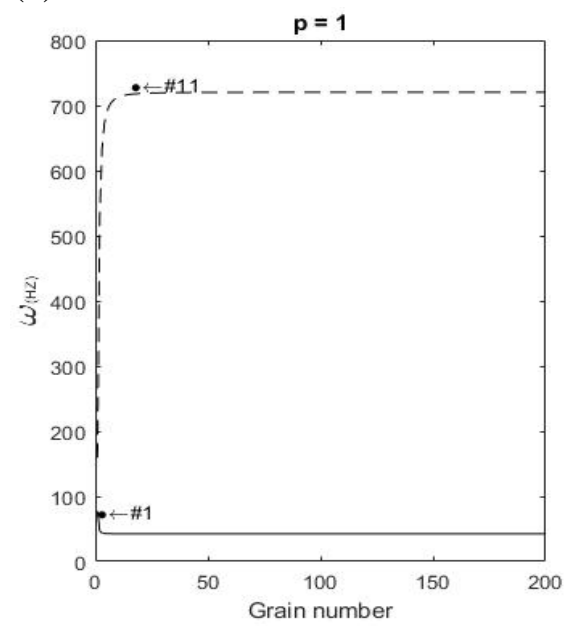

(b)

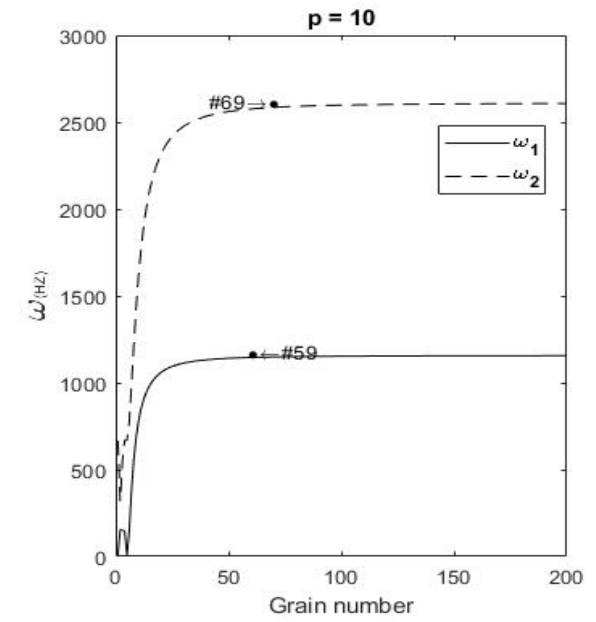

Fig. 8. Analysis of the grain number effect on the frequencies (discrete exact solution) for the mode number (a) $p=1$ and (b) $p=10$ with respect to the length ratio $\left(r^{*}=0.029\right)$ for $\mu_{s}=4.28$ and $k^{*}=1.87$.

\section{Nonlocal Approximate Solutions - Continuous Approach}

The fourth-order difference equations of Eq. (19) may be continualized in two general ways: the simplest approach is based on the polynomial expansions in which the finite differences operators are expanded with the Taylor approximation. This leads to a higher-order gradient Cosserat continuum theory. Another effective method considers a rational expansion based on the Padé approximation which could give better homogenized solution compared to the Taylor series (see for instance Duan et al. [24] for the application of this technique to granular system). The second strategy is based on homogenization of the equations by means of a discrete Fourier transformation. The result, in this case, is a Kunin-type non-local theory.

In the next section, the discrete nature of the granular beam structure which has been modeled utilizing the difference equation as Eq. (19) is continualized by applying the Taylor series and the Padé approximation.

\subsection{Polynomial Expansion (Taylor Series Approximant)}

The general solution for the granular beam will be investigated by a continualization transform based on exponential pseudo-differential operators. The following pseudodifferential operators are defined in order to introduce the relation between the discrete and the equivalent continuous system holds for a sufficiently smooth deflection function (Salvadori [36]):

$$
\begin{gathered}
w_{i}=w(\mathrm{x}=\mathrm{ia}) \\
w_{i+1}=\sum_{k=0}^{\infty} \frac{a^{k} D_{x}^{k}}{k !} w(x)=\left[1+\frac{a D_{x}^{1}}{1 !}+\frac{a^{2} D_{x}^{2}}{2 !}+\frac{a^{3} D_{x}^{3}}{3 !}+\cdots\right] w(x)=e^{a D_{x}} w(x) ; \mathrm{x}=\mathrm{ia}
\end{gathered}
$$

Subsequently, the involved pseudo-differential equations $\delta_{2}{ }^{2} w(x), \delta_{2} w(x)$ and $\delta_{0} w(x)$ may be defined as: 


$$
\begin{aligned}
& \delta_{2}{ }^{2} w(x)=\left(\frac{e^{2 a D_{x}-4 e^{a D_{x}}+6-4 e^{-a D_{x}}+e^{-2 a D_{x}}}}{a^{4}}\right) w(x)=\left(1+\frac{a^{2} D_{x}^{2}}{6}+\frac{a^{4} D_{x}^{4}}{80}+O\left(a^{6} D_{x}^{6}\right)\right) D_{x}^{4} w(x) \\
& \delta_{2} w(x)=\left(\frac{e^{a D_{x}-2+e^{-a D_{x}}}}{a^{2}}\right) w(x)=\left(1+\frac{a^{2} D_{x}^{2}}{12}+\frac{a^{4} D_{x}^{4}}{360}+0\left(a^{6} D_{x}^{6}\right)\right) D_{x}^{2} w(x) \\
& \delta_{0} w(x)=\left(\frac{e^{a D_{x}}+2+e^{-a D_{x}}}{4}\right) w(x)=\left(1+\frac{a^{2} D_{x}^{2}}{4}+\frac{a^{4} D_{x}^{4}}{48}+\mathrm{O}\left(a^{6} D_{x}^{6}\right)\right) w(x)
\end{aligned}
$$

Substitution of the fourth-order expansions of Eq. (98) for the pseudo-differential operators in Eq. (15) leads to

$$
\begin{gathered}
{\left[E I\left(1+\frac{a^{2} D_{x}^{2}}{6}\right) D_{x}^{4}+\left(\rho I \omega^{2}-\frac{k E I}{K_{s} G A}+\frac{E I \rho A \omega^{2}}{K_{s} G A}\right)\left(1+\frac{a^{2} D_{x}^{2}}{12}\right) D_{x}^{2}+\left(k-\rho A \omega^{2}\right)\left(1+\frac{a^{2} D_{x}^{2}}{4}\right)\right.} \\
\left.-\frac{k \rho I \omega^{2}}{K_{s} G A}+\frac{\rho^{2} I A \omega^{4}}{K_{s} G A}\right] w(x)=0
\end{gathered}
$$

Next a gradient-type differential equation could be obtained as follow with multiplication of the last equation by $\left(1-\frac{a^{2} D_{x}^{2}}{3}\right)$ and neglecting higher-order terms in $a^{4}$.

$$
\begin{gathered}
{\left[E I\left(1-\frac{a^{2} D_{x}^{2}}{6}\right) D_{x}^{4}+\left(\rho I \omega^{2}-\frac{k E I}{K_{s} G A}+\frac{E I \rho A \omega^{2}}{K_{s} G A}\right)\left(1-\frac{a^{2} D_{x}^{2}}{4}\right) D_{x}^{2}+\left(k-\rho A \omega^{2}\right)\left(1-\frac{a^{2} D_{x}^{2}}{12}\right)\right.} \\
\left.+\left(-\frac{k \rho I \omega^{2}}{K_{s} G A}+\frac{\rho^{2} I A \omega^{4}}{K_{s} G A}\right)\left(1-\frac{a^{2} D_{x}^{2}}{3}\right)\right] w(x)=0
\end{gathered}
$$

With ignorance of shear effects in the granular system $\left(K_{S} G A \rightarrow \infty\right)$, Eq. (100) could be simplified

$$
E I\left(1-\frac{a^{2} D_{x}^{2}}{6}\right) D_{x}^{4} w(x)+\left(\rho I \omega^{2}\right)\left(1-\frac{a^{2} D_{x}^{2}}{4}\right) D_{x}^{2} w(x)+\left(k-\rho A \omega^{2}\right)\left(1-\frac{a^{2} D_{x}^{2}}{12}\right) w(x)=0
$$

which is equivalent to

$$
E I\left(-\frac{a^{2}}{6} w^{(6)}+w^{(4)}\right)+k\left(w-\frac{a^{2}}{12} w^{\prime \prime}\right)-\rho A \omega^{2}\left(w-\frac{a^{2}}{12} w^{\prime \prime}\right)+\rho I \omega^{2}\left(w^{\prime \prime}-\frac{a^{2}}{4} w^{(4)}\right)=0
$$

The last equation is formally the same as the one obtained for a gradient elasticity Rayleigh beam equation under Pasternak-type foundation. This differential equation can be obtained from the application of the Hamilton principle associated with some connected energy functions. The (definitive positive) potential energy and kinetic energy functions are respectively given by

$$
\begin{aligned}
& W=\int_{0}^{L} \frac{1}{2} E I\left(w^{\prime \prime 2}+\frac{a^{2}}{6}{w^{\prime \prime \prime}}^{2}\right) d x+\int_{0}^{L} \frac{1}{2} k\left(w^{2}+\frac{a^{2}}{12}{w^{\prime}}^{2}\right) d x=0 \\
& T=\int_{0}^{L} \frac{1}{2} \rho A\left(\dot{w}^{2}+\frac{a^{2}}{12} \dot{w}^{\prime 2}\right) d x+\int_{0}^{L} \frac{1}{2} \rho I\left(\dot{w}^{\prime 2}+\frac{a^{2}}{4} \dot{w}^{\prime \prime 2}\right) d x=0
\end{aligned}
$$

Assuming static case $(\omega=0)$, Eq. (101) leads to an equivalent gradient elasticity of EulerBernoulli beam under Pasternak-type foundation

$$
E I\left(-\frac{a^{2}}{6} w^{(6)}+w^{(4)}\right)+k\left(w-\frac{a^{2}}{12} w^{\prime \prime}\right)=0
$$


Going back to Eq. (99) which is an approximation of the discrete model, the higher-order differential equation could be rewritten

$$
\begin{gathered}
{\left[\frac{E I a^{2}}{6} w^{(6)}+\left[E I+\left(\rho I \omega^{2}-\frac{k E I}{K_{S} G A}+\frac{E I \rho A \omega^{2}}{K_{S} G A}\right) \frac{a^{2}}{12}\right] w^{(4)}+\left[\left(\rho I \omega^{2}-\frac{k E I}{K_{S} G A}+\frac{E I \rho A \omega^{2}}{K_{S} G A}\right)+\right.\right.} \\
\left.\left.\left(k-\rho A \omega^{2}\right) \frac{a^{2}}{4}\right] w^{(2)}+\left[\left(k-\rho A \omega^{2}\right)-\frac{k \rho I \omega^{2}}{K_{S} G A}+\frac{\rho^{2} I A \omega^{4}}{K_{S} G A}\right]\right] w=0
\end{gathered}
$$

Eq. (106) has been obtained by Challamel et al. [27] in the static range $(\omega=0)$. For simply supported boundary conditions, the solution of Eq. (106) could be assumed in the following form:

$$
w(x)=\sin \left(\frac{p \pi x}{L}\right)
$$

So, by substituting this fundamental solution in Eq. (106), the natural frequencies of the granular chain may be obtained from solving the following equation:

$$
\begin{gathered}
{\left[\frac{\rho^{2} I}{k_{S} G}\right] \omega^{4}+\left[\left(\rho I+\frac{E I \rho}{k_{s} G}\right) \frac{a^{2}}{12} \times \frac{p^{4} \pi^{4}}{L^{4}}-\left(\rho I+\frac{E I \rho}{k_{S} G}-\rho A \frac{a^{2}}{4}\right) \frac{p^{2} \pi^{2}}{L^{2}}-\left(\rho A+\frac{k I \rho}{k_{S} G A}\right)\right] \omega^{2}+} \\
{\left[-E I \frac{a^{2}}{6} \times \frac{p^{6} \pi^{6}}{L^{6}}+E I \frac{p^{4} \pi^{4}}{L^{4}}-\frac{k E I}{k_{S} G A} \times \frac{a^{2}}{12} \times \frac{p^{4} \pi^{4}}{L^{4}}+\frac{k E I}{k_{S} G A} \times \frac{p^{2} \pi^{2}}{L^{2}}-k \frac{a^{2}}{4} \times \frac{p^{2} \pi^{2}}{L^{2}}+k\right]=0}
\end{gathered}
$$

or in non-dimensional form

$$
\begin{gathered}
\Omega^{4}+\left[\left(\frac{1}{\mu_{s} r^{2}}+\frac{1}{r^{* 2}}\right) \frac{p^{4} \pi^{4}}{12 n^{2}}-\left(\frac{1}{\mu_{s} r^{* 2}}+\frac{1}{r^{* 2}}-\frac{1}{4 \mu_{s} r^{* 4} n^{2}}\right) p^{2} \pi^{2}-\left(\frac{1}{\mu_{s} r^{* 4}}+k^{*}\right)\right] \Omega^{2}+ \\
{\left[\frac{p^{6} \pi^{6}}{6 \mu_{s} r^{* 4} n^{2}}+\frac{p^{4} \pi^{4}}{\mu_{s} r^{* 4}}-\frac{k^{*} p^{4} \pi^{4}}{12 r^{* 2} n^{2}}+\frac{k^{*} p^{2} \pi^{2}}{r^{* 2}}-\frac{k^{*} p^{2} \pi^{2}}{4 \mu_{s} r^{* 4} n^{2}}+\frac{k^{*}}{\mu_{s} r^{* 4}}\right]=0}
\end{gathered}
$$

By solving the Eq. (109) leads to Eq. (80) with the parameters

$$
\begin{gathered}
B=\left(\frac{1}{\mu_{s} r^{* 2}}+\frac{1}{r^{* 2}}\right) \frac{p^{4} \pi^{4}}{12 n^{2}}-\left(\frac{1}{\mu_{s} r^{* 2}}+\frac{1}{r^{* 2}}-\frac{1}{4 \mu_{S} r^{* 4} n^{2}}\right) p^{2} \pi^{2}-\left(\frac{1}{\mu_{s} r^{* 4}}+k^{*}\right), \\
C=\frac{1}{6 \mu_{s} r^{* 4} n^{2}} p^{6} \pi^{6}+\left(\frac{1}{\mu_{s} r^{* 4}}-\frac{k^{*}}{12 r^{* 2} n^{2}}\right) p^{4} \pi^{4}+\left(\frac{k^{*}}{r^{* 2}}-\frac{k^{*}}{4 \mu_{S} r^{* 4} n^{2}}\right) p^{2} \pi^{2}+\frac{k^{*}}{\mu_{s} r^{* 4}}
\end{gathered}
$$

The natural frequencies could be calculated by substituting Eq. (80) in the following equation with respect to the parameters of Eq. (110).

$$
\omega=\frac{\Omega}{L^{2}} \sqrt{\frac{E I}{\rho A}}
$$

For continuum modeling when $n \rightarrow \infty, \gamma$ change to

$$
\gamma=\sqrt{\left(\frac{p^{2} \pi^{2}}{2 \mu_{s} r^{* 2}}\left(1+\mu_{s}\right)+\frac{k^{*}}{2}+\frac{1}{2 \mu_{s} r^{* 4}}\right) \pm \sqrt{\left(\frac{p^{2} \pi^{2}}{2 \mu_{s} r^{* 2}}\left(1+\mu_{s}\right)+\frac{k^{*}}{2}+\frac{1}{2 \mu_{s} r^{* 4}}\right)^{2}-\left(\frac{k^{*}}{\mu_{s} r^{* 4}}+\frac{k^{*} p^{2} \pi^{2}}{r^{* 2}}+\frac{p^{4} \pi^{4}}{\mu_{s} r^{* 4}}\right)}}
$$

Eq. (112) coincides with Eq. (89), which also exactly agrees with the results of Wang and Stephens [15], Cheng and Pantelides [28] and Manevich [16].

In Figure. 9, the natural frequencies of the discrete granular model obtained from the Taylor expansion of pseudo-differential (nonlocal approach) have been compared with the continuum solution for the two branches. The coincidence of the discrete and continuum eigenfrequencies occurs when the ratio of $n / p$ is sufficiently large which can be quantified for the second branch typically 7 and for the first branch by typical value of 5 . In comparison with the exact solution, 
it can be clarified that the Taylor approximation requires more discrete elements in order to converge to the continuum results.

It is important to note that by decreasing the $n / p$ ratio, the imaginary term appears in the nonlocal results for the two branches. For these cases, the real parts of the two branches are equal together while the imaginary parts are equal in values but opposite in sign. Therefore, using the Taylor series for continualizing the difference equations of the granular beam implies imaginary eigenfrequencies. A similar conclusion has been obtained also by Bažant and Christensen [37]. The length ratio and grain number effects on frequencies have been studied in Figure. 10.

(a)

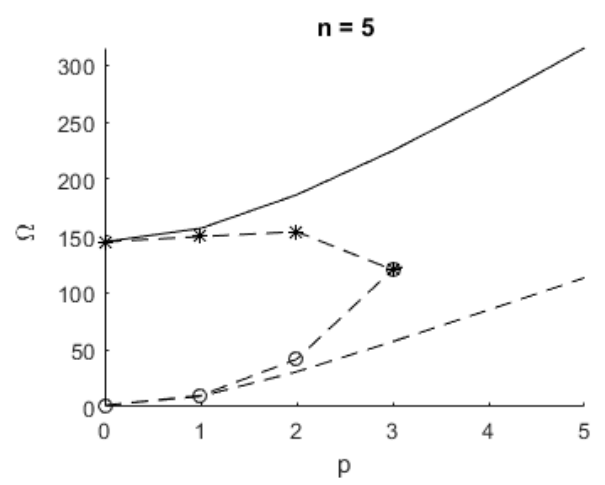

(c)

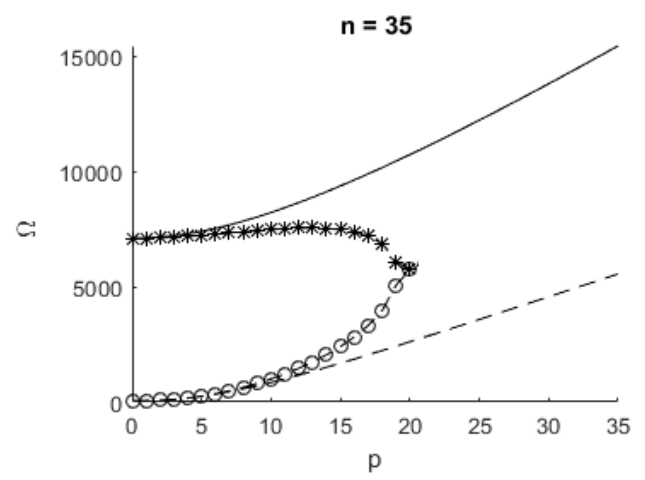

(b)

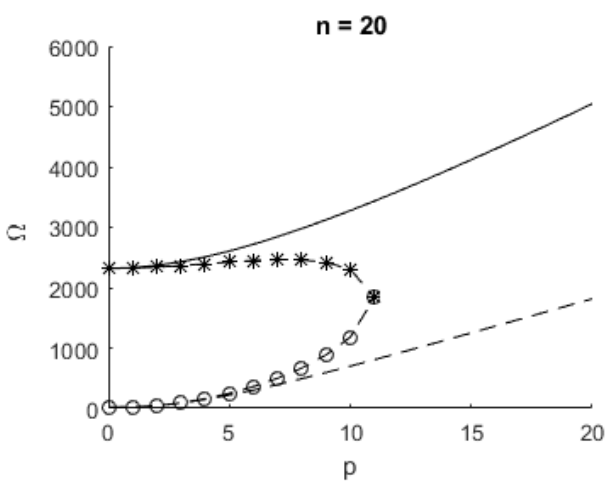

(d)

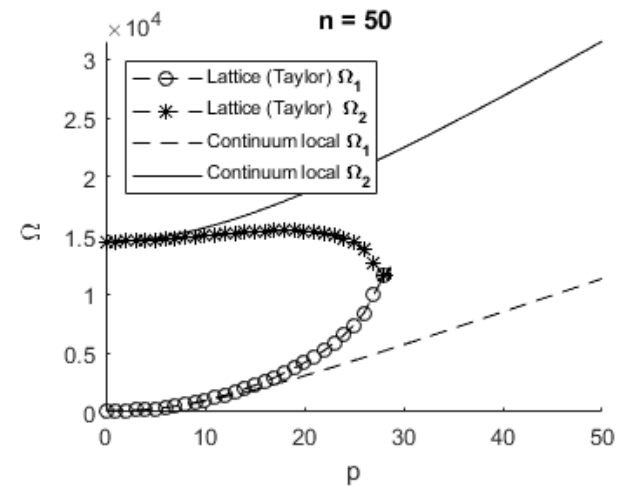

Fig. 9. Comparison of the natural frequencies for the nonlocal Taylor and continuum solutions with respect to the mode number (p) and grain number: (a) $n=5$ and $r^{*}=0.058$, (b) $n=20$ and $r^{*}=0.014$, (c) $n=35$ and $r^{*}=0.0082$ and (d) $n=50$ and $r^{*}=0.0058$ for $\mu_{s}=4.28$.

(a)

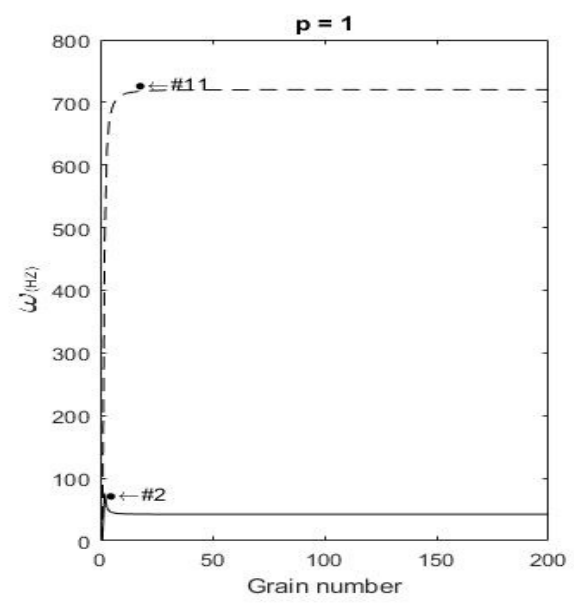

(b)

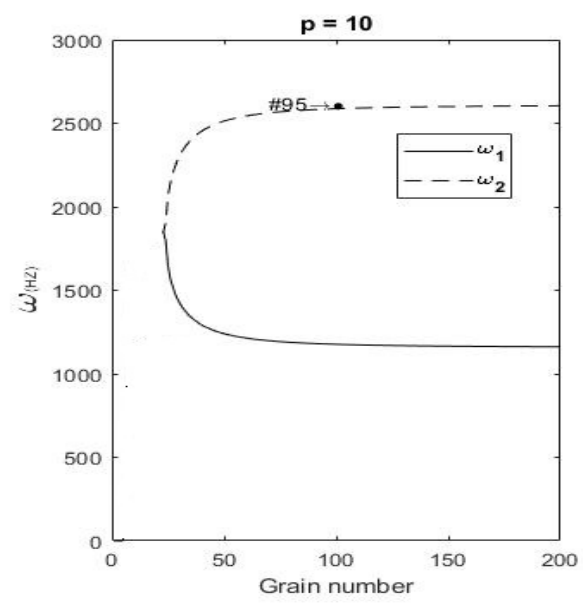


Fig. 10. Analysis of the grain number effect on the frequencies (nonlocal Taylor) for the mode number (a) $p=1$ and (b) $p=10$ with respect to the length ratio for $\mu_{s}=4.28$ and $k^{*}=1.87$.

\subsection{Rational Expansion (Padé Approximant)}

In this section, the approximation of Padé has been used in the asymptotic expansion of the pseudo-differential operators. This method often gives a better approximation of a function than its Taylor series counterpart (Baker and Graves-Morris [38]).

Applying the Padé approximant of [1/4], [1/2] and [1/2] in Eq. (98) yields:

$$
\begin{gathered}
\delta_{2}{ }^{2} w(x) \approx\left(\frac{1}{1-\frac{a^{2} D_{x}^{2}}{6}+\frac{11 a^{4} D_{x}^{4}}{720}}\right) D_{x}^{4} w(x) \approx\left(\frac{1}{\left(1-\frac{a^{2} D_{x}^{2}}{12}\right)^{2}}\right) D_{x}^{4} w(x) \\
\delta_{2} w(x) \approx\left(\frac{1}{1-\frac{a^{2} D_{x}^{2}}{12}}\right) D_{x}^{2} w(x) \\
\delta_{0} w(x) \approx\left[1+\left(\frac{1}{1-\frac{a^{2} D_{x}^{2}}{12}}\right) \frac{a^{2} D_{x}^{2}}{4}\right] w(x)
\end{gathered}
$$

Eq. (15) could be rewritten as a function of Eq. (98)

$$
\begin{gathered}
{\left[\left(\frac{1}{\left(1-\frac{a^{2} D_{x}^{2}}{12}\right)^{2}}\right) D_{x}^{4}+\left(\frac{\rho I}{E I} \omega^{2}-\frac{k}{K_{S} G A}+\frac{\rho A \omega^{2}}{K_{S} G A}\right)\left(\frac{1}{1-\frac{a^{2} D_{x}^{2}}{12}}\right) D_{x}^{2}+\left(\frac{k}{E I}-\frac{\rho A \omega^{2}}{E I}\right)\left(1+\left(\frac{1}{1-\frac{a^{2} D_{x}^{2}}{12}}\right) \frac{a^{2} D_{x}^{2}}{4}\right)+\right.} \\
\left.\left(-\frac{k \rho I \omega^{2}}{E I K_{S} G A}+\frac{\rho^{2} I A \omega^{4}}{E I K_{S} G A}\right)\right] w(x)=0
\end{gathered}
$$

Multiplication of Eq. (114) by $\left(1-\frac{a^{2} D_{x}^{2}}{12}\right)^{2}$ leads to the following compact form equation.

$$
\begin{aligned}
\left(D_{x}^{4}+\left[\frac{\rho \omega^{2}}{E}+\frac{\rho \omega^{2}}{K_{S} G}\right.\right. & \left.-\frac{\rho A \omega^{2}}{4 E I} a^{2}-\frac{k}{K_{S} G A}+\frac{k a^{2}}{4 E I}\right]\left(1-\frac{a^{2} D_{x}^{2}}{12}\right) D_{x}^{2} \\
& \left.+\left[\frac{\rho^{2} \omega^{4}}{E K_{S} G}-\frac{\rho A \omega^{2}}{E I}-\frac{k \rho \omega^{2}}{E K_{S} G A}+\frac{k}{E I}\right]\left(1-\frac{a^{2} D_{x}^{2}}{12}\right)^{2}\right) w_{(x)}=0
\end{aligned}
$$

Eq. (115) can be written in the dimensionless form

$$
\left(L^{4} D_{x}^{4}+\alpha\left(1-\frac{a^{2} D_{x}^{2}}{12}\right) L^{2} D_{x}^{2}+\beta\left(1-\frac{a^{2} D_{x}^{2}}{6}+\frac{a^{4} D_{x}^{4}}{144}\right)\right) w(x)=0
$$

in which $\alpha$ and $\beta$ are defined as:

$$
\alpha=\left[r^{* 2} \Omega^{2}\left(1+\mu_{s}-\frac{1}{4 r^{* 2} n^{2}}\right)-r^{* 2} k^{*} \mu_{s}+\frac{k^{*}}{4 n^{2}}\right], \beta=-\left[r^{* 2} \Omega^{2}\left(\mu_{s} r^{* 2} k^{*}+\frac{1}{r^{* 2}}-\mu_{s} r^{* 2} \Omega^{2}\right)-k^{*}\right]
$$

Using Eq. (113), the dimensionless differential equation can be presented in the following form:

$$
\begin{gathered}
{\left[\frac{r^{* 2} \Omega^{2}}{144 n^{4}}\left(\mu_{s} r^{* 2} \Omega^{2}-\mu_{s} r^{* 2} k^{*}-\frac{1}{r^{* 2}}\right)-\frac{r^{* 2} \Omega^{2}}{12 n^{2}}\left(1+\mu_{s}-\frac{1}{4 r^{* 2} n^{2}}\right)+\frac{r^{* 2} k^{*} \mu_{S}}{12 n^{2}}-\frac{k^{*}}{48 n^{4}}+\frac{k^{*}}{144 n^{4}}+\right.} \\
1] \frac{d^{4} \bar{w}}{d \bar{x}^{4}}+\left[r^{* 2} \Omega^{2}\left(1+\mu_{s}-\frac{1}{4 r^{* 2} n^{2}}\right)-r^{* 2} k^{*} \mu_{s}+\frac{k^{*}}{4 n^{2}}+\frac{r^{* 2} \Omega^{2}}{6 n^{2}}\left(\mu_{s} r^{* 2} k^{*}+\frac{1}{r^{* 2}}-\mu_{s} r^{* 2} \Omega^{2}\right)-\right. \\
\left.\frac{k^{*}}{6 n^{2}}\right] \frac{d^{2} \bar{w}}{d \bar{x}^{2}}+\left[r^{* 2} \Omega^{2}\left(\mu_{s} r^{* 2} \Omega^{2}-\mu_{s} r^{* 2} k^{*}-\frac{1}{r^{* 2}}\right)+k^{*}\right] \bar{w}=0
\end{gathered}
$$

Ignoring Winkler foundation effect $\left(k^{*}=0\right)$, Eq. (118) leads to 


$$
\begin{gathered}
{\left[\frac{r^{* 2} \Omega^{2}}{144 n^{4}}\left(\mu_{s} r^{* 2} \Omega^{2}-\frac{1}{r^{* 2}}\right)-\frac{r^{* 2} \Omega^{2}}{12 n^{2}}\left(1+\mu_{s}-\frac{1}{4 r^{* 2} n^{2}}\right)+1\right] \frac{d^{4} \bar{w}}{d \bar{x}^{4}}+\left[r^{* 2} \Omega^{2}\left(1+\mu_{s}-\frac{1}{4 r^{* 2} n^{2}}\right)+\right.} \\
\left.\frac{r^{* 2} \Omega^{2}}{6 n^{2}}\left(\frac{1}{r^{* 2}}-\mu_{s} r^{* 2} \Omega^{2}\right)\right] \frac{d^{2} \bar{w}}{d \bar{x}^{2}}+\left[r^{* 2} \Omega^{2}\left(\mu_{s} r^{* 2} \Omega^{2}-\frac{1}{r^{* 2}}\right)\right] \bar{w}=0
\end{gathered}
$$

If the pseudo-differential operator effect of $\delta_{0} w(x)$ is neglected, Eq. (119) leads to the one of Duan et al. [24]. The dimensionless differential equation of Duan et al. [24] is given by

$$
\begin{gathered}
{\left[\frac{r^{* 2} \Omega^{2}}{144 n^{4}}\left(\mu_{s} r^{* 2} \Omega^{2}-\frac{1}{r^{* 2}}\right)-\frac{r^{* 2} \Omega^{2}}{12 n^{2}}\left(1+\mu_{s}\right)+1\right] \frac{d^{4} \bar{w}}{d \bar{x}^{4}}+\left[r^{* 2} \Omega^{2}\left(1+\mu_{s}\right)+\frac{r^{* 2} \Omega^{2}}{6 n^{2}}\left(\frac{1}{r^{* 2}}-\right.\right.} \\
\left.\left.\mu_{s} r^{* 2} \Omega^{2}\right)\right] \frac{d^{2} \bar{w}}{d \bar{x}^{2}}+\left[r^{* 2} \Omega^{2}\left(\mu_{s} r^{* 2} \Omega^{2}-\frac{1}{r^{* 2}}\right)\right] \bar{w}=0
\end{gathered}
$$

For the simply supported boundary conditions the solution of the Eq. (118) can be considered again as the form of Eq. (92). Substitution of the Eq. (92) in Eq. (118) yields:

$$
\begin{gathered}
{\left[\frac{\mu_{s} r^{* 4} p^{4} \pi^{4}}{144 n^{4}}+\frac{\mu_{s} r^{* 4} p^{2} \pi^{2}}{6 n^{2}}+\mu_{s} r^{* 4}\right] \Omega^{4}+\left[\frac{r^{* 2} p^{4} \pi^{4}}{144 n^{4}}\left(-\mu_{s} r^{* 2} k^{*}-\frac{1}{r^{* 2}}\right)-\frac{r^{* 2} p^{4} \pi^{4}}{12 n^{2}}\left(1+\mu_{s}-\right.\right.} \\
\left.\left.\frac{1}{4 r^{* 2} n^{2}}\right)-r^{* 2} p^{2} \pi^{2}\left(1+\mu_{s}-\frac{1}{4 r^{* 2} n^{2}}\right)-\frac{r^{* 2} p^{2} \pi^{2}}{6 n^{2}}\left(r^{* 2} \mu_{s} k^{*}+\frac{1}{r^{* 2}}\right)+r^{* 2}\left(-\mu_{s} r^{* 2} k^{*}-\frac{1}{r^{* 2}}\right)\right] \Omega^{2}+ \\
{\left[\frac{r^{* 2} k^{*} \mu_{s} p^{4} \pi^{4}}{12 n^{2}}-\frac{k^{*} p^{4} \pi^{4}}{48 n^{4}}+\frac{k^{*} p^{4} \pi^{4}}{144 n^{4}}+p^{4} \pi^{4}+r^{* 2} k^{*} \mu_{s} p^{2} \pi^{2}-\frac{k^{*} p^{2} \pi^{2}}{4 n^{2}}+\frac{k^{*} p^{2} \pi^{2}}{6 n^{2}}+k^{*}\right]=0}
\end{gathered}
$$

or in the compact form

$$
A \Omega^{4}-B \Omega^{2}+C=0
$$

$A, B$ and $C$ are defined as:

$$
\begin{gathered}
A=\frac{\mu_{s} r^{* 4} p^{4} \pi^{4}}{144 n^{4}}+\frac{\mu_{S} r^{* 4} p^{2} \pi^{2}}{6 n^{2}}+r^{* 4} \mu_{S} \\
B=\frac{r^{* 2} p^{4} \pi^{4}}{144 n^{4}}\left(\mu_{s} r^{* 2} k^{*}+\frac{1}{r^{* 2}}\right)+\left(\frac{r^{* 2} p^{4} \pi^{4}}{12 n^{2}}+r^{* 2} p^{2} \pi^{2}\right)\left(1+\mu_{s}-\frac{1}{4 r^{* 2} n^{2}}\right)+\left(\frac{r^{* 2} p^{2} \pi^{2}}{6 n^{2}}+\right. \\
\left.r^{* 2}\right)\left(\mu_{s} r^{* 2} k^{*}+\frac{1}{r^{* 2}}\right), \\
C=\frac{k^{*} p^{2} \pi^{2}}{12 n^{2}}\left(r^{* 2} \mu_{s} p^{2} \pi^{2}-\frac{p^{2} \pi^{2}}{6 n^{2}}-1\right)+p^{4} \pi^{4}+r^{* 2} k^{*} \mu_{S} p^{2} \pi^{2}+k^{*}
\end{gathered}
$$

where $p$ is the mode number (natural number). $\Omega$ would be obtained by

$$
\Omega=\sqrt{\frac{B \pm \sqrt{B^{2}-4 A C}}{2 A}}
$$

For continuum case when $n \rightarrow \infty$, Eq. (121) could be written in a quartic form

$$
\Omega^{4}-B \Omega^{2}+C=0
$$

$B$ and $C$ are defined as:

$$
B=\frac{p^{2} \pi^{2}}{\mu_{S} r^{* 2}}\left(1+\mu_{S}\right)+\frac{1}{\mu_{S} r^{* 2}}\left(\mu_{S} r^{* 2} k^{*}+\frac{1}{r^{* 2}}\right), \quad C=\frac{p^{4} \pi^{4}}{\mu_{S} r^{* 4}}+\frac{k^{*} p^{2} \pi^{2}}{r^{* 2}}+\frac{k^{*}}{\mu_{S} r^{* 4}}
$$

Thus, again the natural frequency could be obtained by Eq. (81) with $\gamma$ expressed

$$
\gamma=\sqrt{\frac{p^{2} \pi^{2}}{2 \mu_{s} r^{* 2}}\left(1+\mu_{s}\right)+\frac{k^{*}}{2}+\frac{1}{2 \mu_{s} r^{* 4}} \pm \sqrt{\left(\frac{p^{2} \pi^{2}}{2 \mu_{s} r^{* 2}}\left(1+\mu_{s}\right)+\frac{k^{*}}{2}+\frac{1}{2 \mu_{s} r^{* 4}}\right)^{2}-\left(\frac{k^{*}}{\mu_{s} r^{* 4}}+\frac{k^{*} p^{2} \pi^{2}}{r^{* 2}}+\frac{p^{4} \pi^{4}}{\mu_{s} r^{* 4}}\right)}}
$$


The last equation is valid for the continuum case and agrees with the results of the BresseTimoshenko beam on an elastic foundation of Winkler and also Wang and Stephens [15], Cheng and Pantelides [28] and Manevich [16].

The natural frequencies of the two existed branches regarding Eq. (127), have been shown in Figure. 11. The frequencies obtained by the Padé approximants can be supposed equal to the continuum ones when the ratio of $n / p$ is large enough. For the second branch, this ratio needs to be typically higher than 5 while for the first branch this limit value is typically 3 . These typical limit values are the same as ones obtained by the exact solution. Figure. 12 shows the length ratio $\left(r^{*}\right)$ and grain number effects on frequencies. Increasing the length ratio (refers to beam thickness/beam length) causes an increase in the eigenfrequencies. The values of grain number limit $(n *)$ have been also reported for each case.

(a)

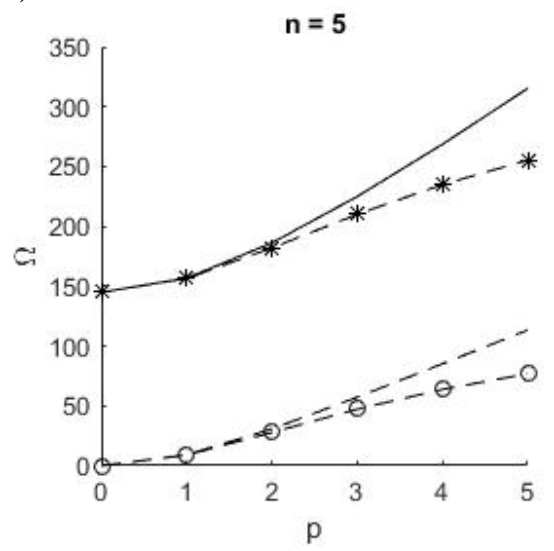

(c)

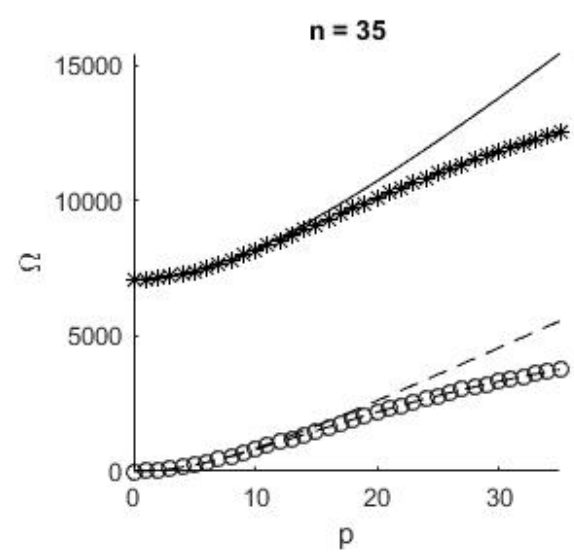

(b)

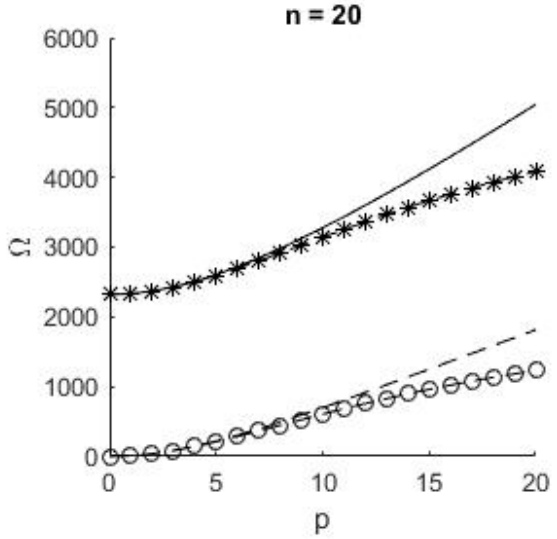

(d)

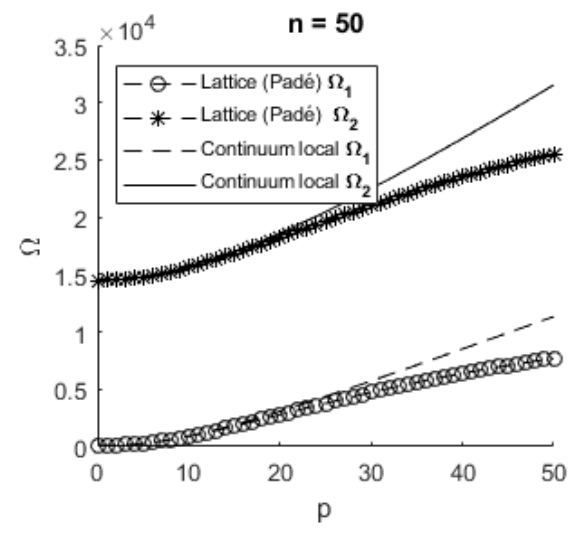

Fig. 11. Comparison of the natural frequencies for the nonlocal Padé and continuum solutions with respect to the mode number (p) and grain number: (a) $n=5$ and $r^{*}=0.058$, (b) $n=20$ and $r^{*}=0.014$, (c) $n=35$ and $r^{*}=0.0082$ and (d) $n=50$ and $r^{*}=0.0058$ for $\mu_{s}=4.28$.

(a)

(b) 

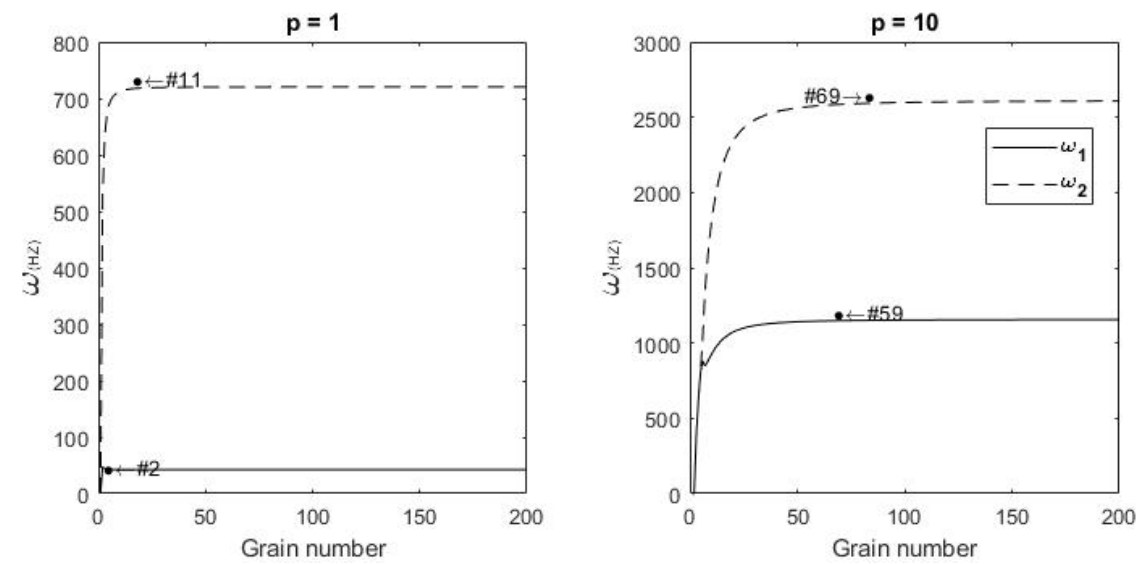

Fig. 12. Analysis of the grain number effect on the frequencies (nonlocal Padé) with respect to the length ratio $\left(r^{*}=\right.$ 0.029 ) for mode number: (a) $p=1$ and (b) $p=10 \mu_{s}=4.28$.

\section{Discussion}

The eigenfrequency results of the two branches are gathered together for all approaches (local, nonlocal and continuum ones) in Figure. 13 and Figure. 14. The results are reported as a function of mode number $(p)$ for four typical grain number values $(n \in\{5,20,35,50\})$ and the dimensionless parameters of $r^{*} \in\{0.058,0.014,0.0082,0.0058\}$ and $k^{*} \in$ $\{1.875,480,4502,18750\}$. It can be obtained from the figures that the results of Padé approximation are closer to the ones obtained by exact resolution. Another point is that the eigenfrequencies obtained by the Taylor series are imaginary when the ratio of $n / p$ is less than the typical approximate value of 2 . Furthermore, the results are investigated for the weak shear interaction $\left(K_{S} G A \rightarrow 0\right)$ model in Figure. 15 and Figure. 16.

(a)

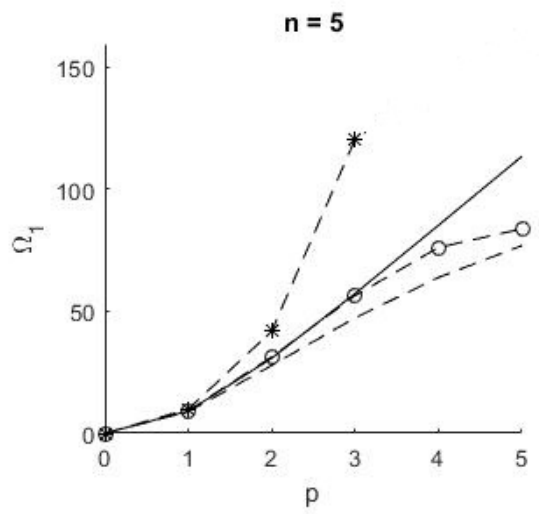

(c)

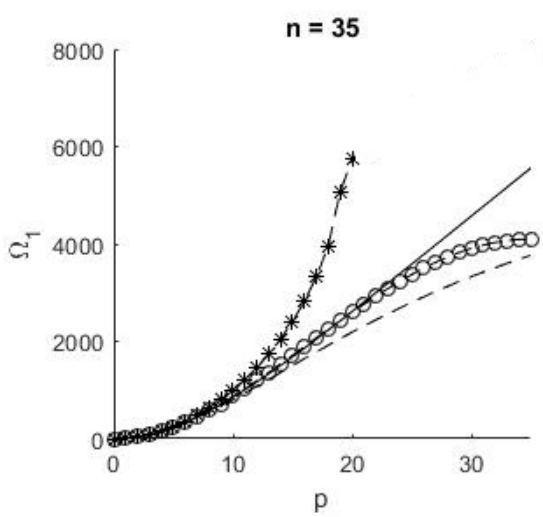

(b)

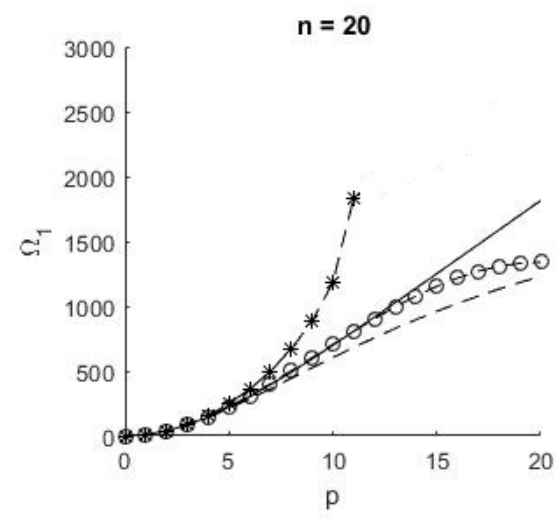

(d)

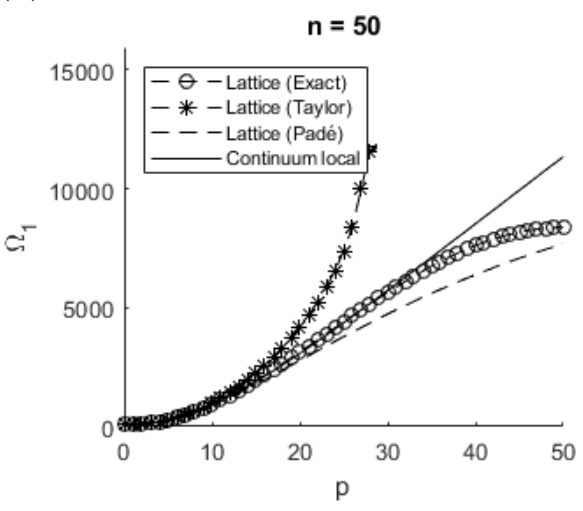


Fig. 13. Comparison of the first branch natural frequencies for different approaches as a function of mode number (p) with respect to the grain number: (a) $n=5$, (b) $n=20$, (c) $n=35$ and (d) $n=50$ for $\mu_{s}=4.28$.

(a)

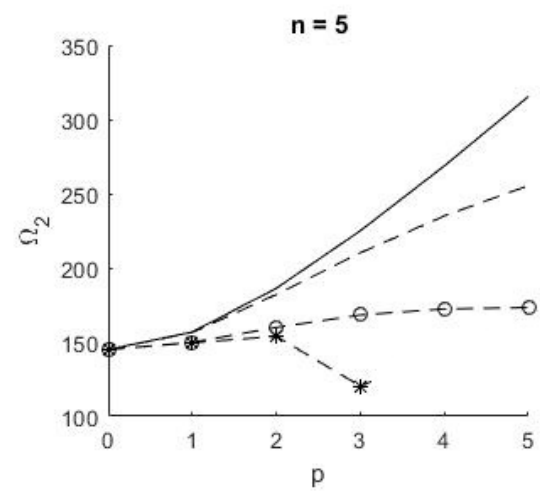

(c)

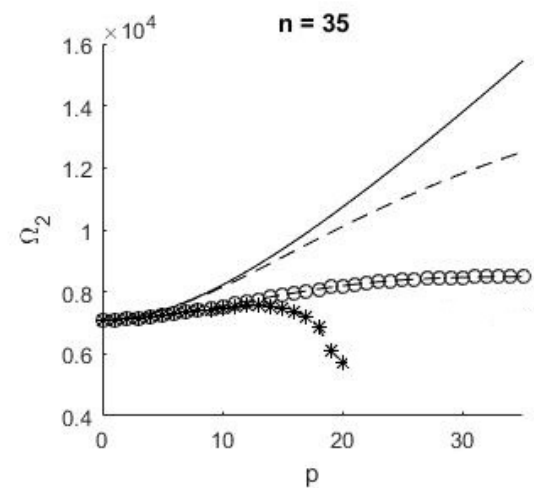

(b)

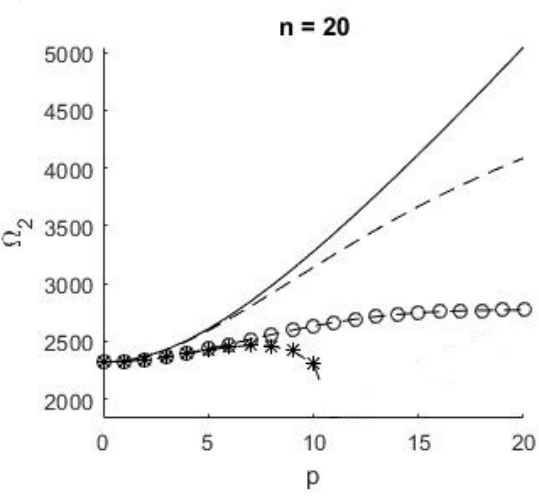

(d)

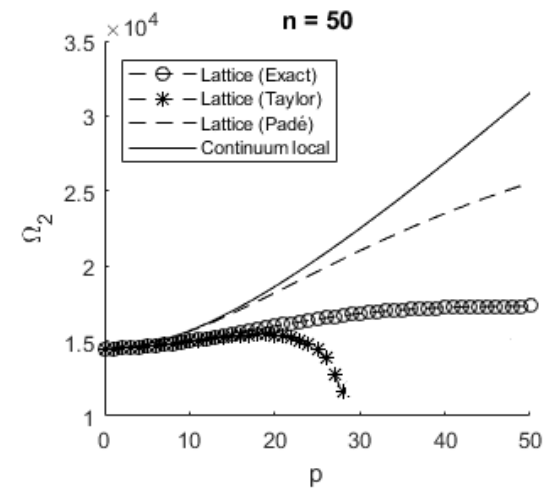

Fig. 14. Comparison of the second branch natural frequencies for different approaches as a function of mode number (p) with respect to the grain number: (a) $n=5$ and $r^{*}=0.058$, (b) $n=20$ and $r^{*}=0.014$, (c) $n=35$ and $r^{*}=$ 0.0082 and (d) $n=50$ and $r^{*}=0.0058$ for $\mu_{s}=4.28$.

(a)

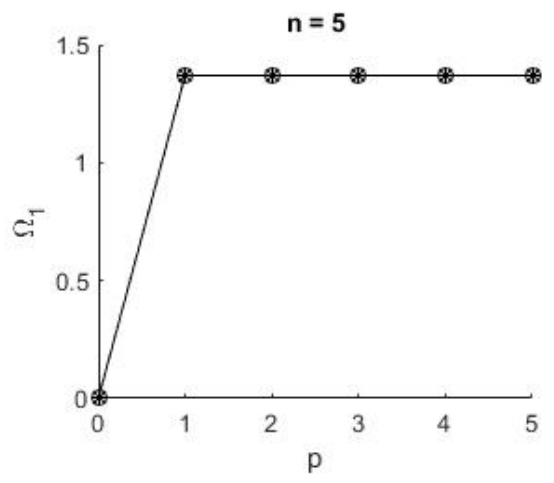

(c) (b)

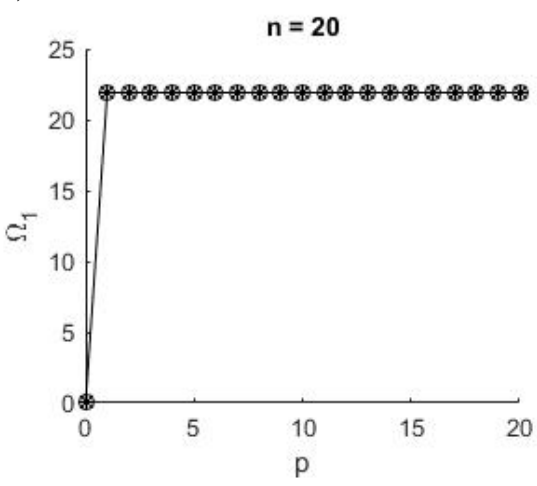

(d) 

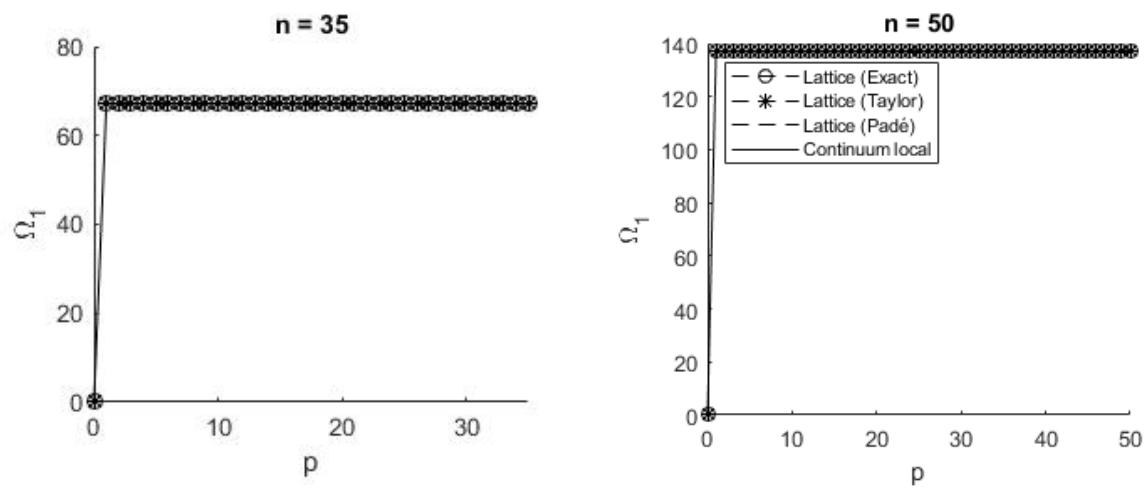

Fig. 15. Comparison of the natural frequencies of the first branch for different approaches as a function of mode number (p) with respect to the grain number: (a) $n=5$ and $r^{*}=0.058$, (b) $n=20$ and $r^{*}=0.014$, (c) $n=35$ and $r^{*}=$ 0.0082 and (d) $n=50$ and $r^{*}=0.0058$ for $\mu_{s} \rightarrow \infty$ (weak shear interaction).

(a)

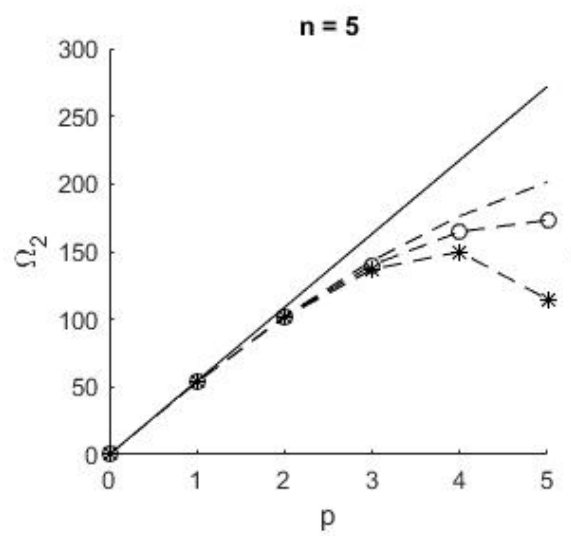

(c)

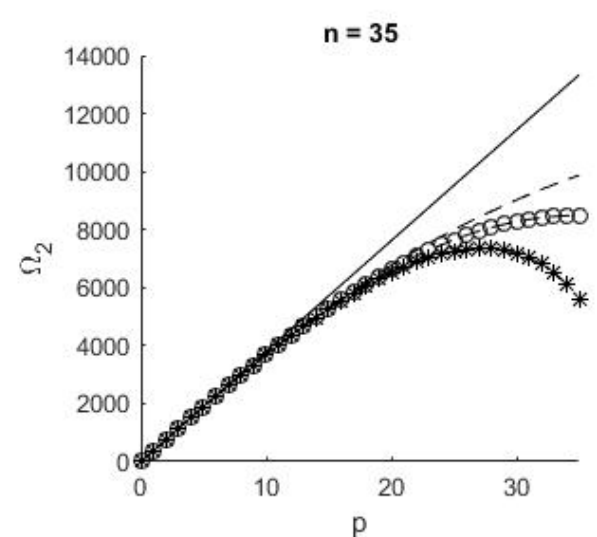

(b)

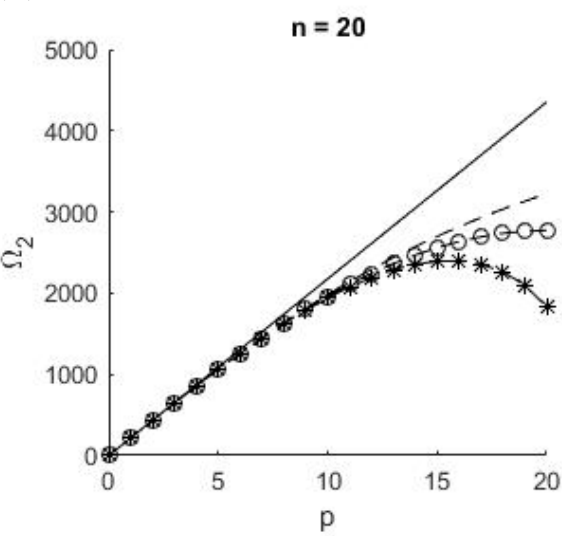

(d)

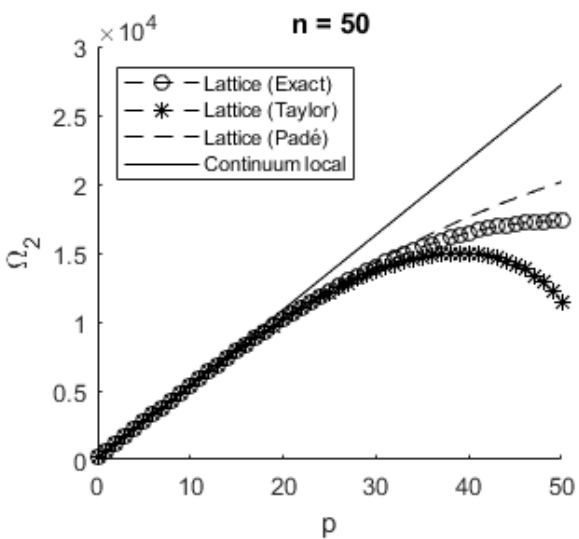

Fig. 16. Comparison of the natural frequencies of the second branch for different approaches as a function of mode number (p) with respect to the grain number: (a) $n=5$ and $r^{*}=0.058$, (b) $n=20$ and $r^{*}=0.014$, (c) $n=35$ and $r^{*}=0.0082$ and (d) $n=50$ and $r^{*}=0.0058$ for $\mu_{s} \rightarrow \infty$ (weak shear interaction).

The results of $\Omega_{10} / \Omega_{0}$ on a Winkler type foundation for the first five modes, with length ratio $\left(r^{*}\right)$ varying from 0 to 0.1 are plotted in Figure. 17. 


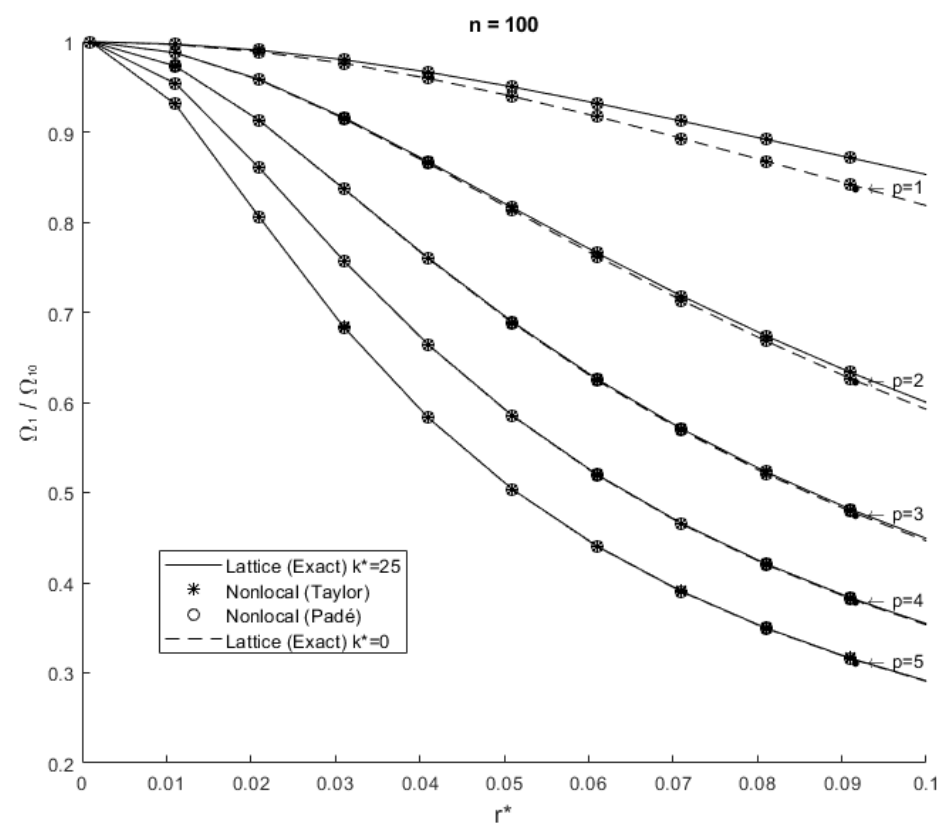

Fig. 17. Correction in the first branch natural frequencies regarding to Winkler foundation effect.

\section{Conclusion}

This paper investigates the macroscopic free vibration behavior of a discrete granular system resting on a Winkler elastic foundation. This microstructured system consists of uniform grains elastically connected by shear and rotation springs. It is shown that the discrete deflection equation of this granular system (Cosserat chain) is mathematically equivalent to the finite difference formulation of a shear deformable Bresse-Timoshenko beam resting on Winkler foundation. Next, the natural frequencies of such a granular model with simply supported ends are first analytically investigated, whatever considered modes through the resolution of a linear difference equation.

The model is continualized to its equivalent continuous system by using two approximate methods based on the Taylor series and Padé approximants (nonlocal continuum). The eigenfrequencies obtained from the continualized beam using the Padé approximation has shown a good performance if compared to the corresponding one from Taylor approximation. Nevertheless, it has been shown that in some cases the approach based on the Taylor approximant provides imaginary values for the two eigenfrequencies branches without physical sense for the homogenized continuum.

The dependency of the beam dynamic responses to its length ratio is clarified and the equations of the eigenfrequencies are obtained regarding the discrete Cosserat model, local and nonlocal continuous ones. Finally, the results of the exact approach for discrete Cosserat model are compared with those of the nonlocal continuous approach. It is found that the shear stiffness (represented by shear springs) has a significant effect on the vibration frequencies. Furthermore, the scale effects of the granular chain are captured by the continuous gradient elasticity model. This scale effect is related to the grain size with respect to the total length of the Cosserat chain.

\section{References}

[1] E. Cosserat and F. Cosserat, Theories of the deformable bodies, A. Herrmann et Fils, Paris, 1909. 
[2] W. Nowacki, The linear theory of micropolar elasticity, W. Nowacki and W. Olszak (eds.), Micropolar Elasticity. Wien, New-York (Springer-Verlag) (1974) 1-43.

[3] W. Voigt, Theoritical studies on the elasticity relationships of cristals, Abh. Gesch. Wissenschaften, Göttingen, 1887.

[4] A. Eringen, Microcontinuum field theories, Springer, New York, 1999.

[5] A. Eringen, Nonlocal continuum field theories, Springer, New York, 2002.

[6] S. Forest, Generalized continua, In: Buschow, K., Cahn, R., Flemings, M., Ilschner, B., Kramer, E., Mahajan, S. (Eds.), Encyclopedia of Materials: Science and Technology Updates, Elsevier, 2005.

[7] S. Feng, Percolation properties of granular elastic networks in two dimensions, Physical Review B 32 (1) (1985) 510-513. https://journals.aps.org/prb/abstract/10.1103/PhysRevB.32.510

[8] L. M. Schwartz, D. L. Johnson and S. Feng, Vibrational modes in granular materials, Physical Review 52 (10) (1984) 831-834. https://journals.aps.org/prl/abstract/10.1103/PhysRevLett.52.831

[9] J. A. C. Bresse, Cours de mécanique appliquée-Résistance des matériaux et stabilité des constructions GautierVillars, Paris, 1859.

[10] S. P. Timoshenko, On the correction for shear of the differential equation for transverse vibrations of prismatic bars, Philosophical Magazine 41 (1921) 744-746.

[11] S. P. Timoshenko, On the transverse vibration of bars with uniform cross-section, Philosophical Magazine 43 (1922) 125-131.

[12] M. Ostoja-Starzewski, Lattice models in micromechanics, Journal of Applied Mechanics 55 (1) (2002) 3560. https://asmedigitalcollection.asme.org/appliedmechanicsreviews/article-abstract/55/1/35/458978

[13] M. Attar, A. Karrech and K. Regenauer-Lieb, Free vibration analysis of a cracked shear deformable beam on a two-parameter elastic foundation using a lattice spring model, Journal of Sound and Vibration 333 (2014) 23592377. https://www.sciencedirect.com/science/article/abs/pii/S0022460X13009267

[14] A. A. Vasiliev, A. E. Miroshnichenko and M. Ruzzene, A discrete model and analysis of one-dimensional deformations in a structural interface with micro-rotations, mechanics Research Communications 37 (2) (2010) 225-229. https://www.sciencedirect.com/science/article/abs/pii/S0093641309001669

[15] T. M. Wang and J. E. Stephens, Natural frequencies of Timoshenko beams on Pasternak foundations, Journal of Sound and Vibration 51 (2) (1977) 149-155.

https://www.sciencedirect.com/science/article/abs/pii/S0022460X77800291

[16] A. I. Manevich, Dynamics of Timoshenko beam on linear and nonlinear foundation: phase relations, significance of the second spectrum, stability, Journal of Sound and Vibration 344 (2015) 209-220. https://www.sciencedirect.com/science/article/abs/pii/S0022460X15000735

[17] I. Elishakoff, G. M. Tonzani and A. Marzani, Three alternative versions of Bresse-Timoshenko theory for beam, International Journal of Mechanic Science 149 (2018) 402-412.

https://www.sciencedirect.com/science/article/pii/S0020740317302278

[18] I. Elishakoff, Handbook on Timoshenko-Ehrenfest and Uflyand-Mindlin plate theories, World Scientific Publishing Company, 2019.

[19] N. Challamel and I. Elishakoff, A brief history of first-order shear-deformable beam and plate models, mechanics Research Communications 102 (103389) (2019) 1-8.

https://www.sciencedirect.com/science/article/abs/pii/S0093641319302289

[20] M. B. Rubin, On the quest for the best Timoshenko shear coefficient, Journal of Applied Mechanics 70 (2003) 154-158. https://asmedigitalcollection.asme.org/appliedmechanics/article-abstract/70/1/154/447384/On-theQuest-for-the-Best-Timoshenko-Shear?redirectedFrom=fulltext

[21] G. Exadaktylos, Overview of micro-elasticity theories with emphasis on strain gradient elasticity: part I Theoretical considerations, CTU, Prague, 2017.

[22] E. Winkler, The doctrine of elasticity and strength, Dominicus, Prague, 1867. 
[23] E. Pasternak and H. B. Mühlhaus, Generalized homogenization procedures for granular materials, Journal of Engineering Mathematics 51 (1) (2005) 199-229. https://link.springer.com/article/10.1007/s10665-004-3950-z

[24] W. H. Duan, N. Challamel, C. M. Wang and Z. Ding, Development of analytical vibration solutions for microstructured beam model to calibrate length scale coefficient in nonlocal Timoshenko beams, Journal of Applied Physics 114 (2013) 104312-104323. https://aip.scitation.org/doi/10.1063/1.4820565

[25] A. Bacigalupo and C. Gambarotta, Generalized micropolar continualization of 1D beam lattices, Journal of Mechanic Science 155 (2019) 554-570. https://www.semanticscholar.org/paper/Generalized-micropolarcontinualization-of-1D-beam-Bacigalupo-Gambarotta/cdfa099a77c74e0b898f3c4e8c7f9d763a8f0986

[26] N. Challamel, J. Lerbet and C. M. Wang, On buckling of granular columns with shear interaction: discrete versus nonlocal approaches, Journal of Applied Physics 115 (2014) 234902.

https://aip.scitation.org/doi/abs/10.1063/1.4883540

[27] N. Challamel, J. Lerbet, F. Darve and F. Nicot, Buckling of granular systems with discrete and gradient elasticity Cosserat continua, in preparation, (2019)

[28] F. Y. Cheng and C. P. Pantelides, Dynamic Timoshenko beam-columns on elastic media, Journal of Structure Engineering $114 \quad$ (1988) 1524-1550. https://ascelibrary.org/doi/10.1061/\%28ASCE\%2907339445\%281988\%29114\%3A7\%281524\%29

[29] S. Goldberg, Introduction to difference equations: with illustrative examples from economics, psychology, and sociology, Dover publications, New-York, 1958.

[30] S. Elaydi, An introduction to difference equations, Springer, New York, 2005.

[31] I. Elishakoff and R. Santoro, Error in the finite difference based probabilistic dynamic analysis: analytical evaluation, Journal of Sound and Vibration 281 (2005) 1195-1206.

https://www.researchgate.net/publication/238951362_Error_in_the_finite_difference_based_probabilistic_dyna mic_analysis_Analytical_evaluation

[32] I. Elishakoff and R. Santoro, Accuracy of the finite difference method in stochastic setting, Journal of Sound and Vibration 2911 (2006) 275-284. https:/www.sciencedirect.com/science/article/abs/pii/S0022460X05004049

[33] G. W. Hunt, A. Tordesillas, S. C. Green and J. Shi, Force-chain buckling in granular media: a structural mechanics perspective, Philos Trans A Math Eng Sci 368 (2010) 249-262.

https://www.ncbi.nlm.nih.gov/pubmed/19948554

[34] Z. Zhang, C. M. Wang, N. Challamel and I. Elishakoff, Obtaining Eringen's length scale coefficient for vibrating nonlocal beams via continualization method, Journal of Sound and Vibration 333 (2014) 4977-4990. https://www.sciencedirect.com/science/article/abs/pii/S0022460X1400371X

[35] R. W. Traill-Nash and A. R. Collar, The effects of shear flexibility and rotory inertia of the bending vibrations of beams, Journal of Mechanics and Applied Math 6 (2) (1953) 186-222.

https://academic.oup.com/qjmam/article-abstract/6/2/186/1823872?redirectedFrom=fulltext

[36] M. G. Salvadori, Numerical computation of buckling loads by finite differences, ASCE 116 (1951) 590-624. https://www.semanticscholar.org/paper/Closure-of-\%22Numerical-Computation-of-Buckling-LoadsSalvadori/ffdb1fa7e0721241a49968b54e74fae5ed518eb1

[37] Z. P. Bažant and M. Christensen, Analogy between micropolar continuum and grid frame- works under initial stress, Journal of Solids Structure 8 (1972) 327-346.

https://www.sciencedirect.com/science/article/abs/pii/0020768372900935

[38] A. G. Baker and P. Graves-Morris, Pade approximants, Cambridge University Press, 1996. 